\title{
Quantum interference terms in non-mesonic weak decay of $\Lambda$-hypernuclei within an RPA formalism
}

\author{
E. Bauer ${ }^{a, b, *}$ \\ ${ }^{a}$ Departamento de Física, Universidad Nacional de La Plata, C.C. 67, 1900 La Plata, Argentina \\ b Instituto de Física La Plata, CONICET, 1900 La Plata, Argentina
}

Received 30 July 2007; received in revised form 14 September 2007; accepted 19 September 2007

Available online 21 September 2007

\begin{abstract}
Single and double coincidence nucleon spectra in the $\Lambda$-hypernuclei weak decay are evaluated and discussed using a microscopic formalism. Nuclear matter is employed together with the local density approximation which allows us to analyze the ${ }_{\Lambda}^{12} \mathrm{C}$ hypernucleus non-mesonic weak decay. Final state interactions (FSI) are included via the first order (in the nuclear residual interaction) terms to the RPA, where the strong residual interaction is modelled by a Bonn potential. At this level of approximation, these FSI are pure quantum interference terms between the primary decay $(\Lambda N \rightarrow N N)$ and $(\Lambda N \rightarrow N N \rightarrow N N)$, where the strong interaction is responsible for the last piece in the second reaction. Also the Pauli exchange contributions are explicitly evaluated. We show that the inclusion of Pauli exchange terms is important. A comparison with data is made. We conclude that the limitations in phase space in the RPA make this approximation inadequate to reproduce the nucleon spectra. This fact does not allow us to draw a definite conclusion about the importance of the interference terms.
\end{abstract}

(C) 2007 Elsevier B.V. All rights reserved.

PACS: $21.80 .+\mathrm{a} ; 25.80 . \mathrm{Pw}$

Keywords: $\Lambda$-hypernuclei; Non-mesonic decay of hypernuclei; $\Gamma_{n} / \Gamma_{p}$ ratio

\footnotetext{
* Correspondence address: Departamento de Física, Universidad Nacional de La Plata, C.C. 67, 1900 La Plata, Argentina.

E-mail address: bauer@ fisica.unlp.edu.ar.
} 


\section{Introduction}

A hypernucleus is a bound system of non-strange and strange baryons and it is an important source of information about baryon-baryon strangeness-changing weak interactions. In the present contribution, we focus on the $\Lambda$-hypernuclei, which can be produced by a hadronic reaction—such as $(\pi, K)$ - or by an electromagnetic one-such as $\left(e, e^{\prime} K\right)$. The $\Lambda$ is generally formed in an excited state and then, via the emission of a series of $\gamma$-rays, it reaches its ground $\left(1 s_{1 / 2}\right)$-state. The $\Lambda$ decays via the weak interaction mainly by two decay mechanisms: the socalled mesonic decay $(\Lambda \rightarrow \pi N)$ and the non-mesonic one (NM), where no meson is present in the final state $(\Lambda N \rightarrow n N)$. The mesonic decay is the main decay process for the free $\Lambda$, but when the $\Lambda$ is within the nuclear environment it is strongly inhibited by the Pauli principle. In this case, the non-mesonic weak decay becomes the most important decay mechanism. This decay can be stimulated either by protons or neutrons, where the corresponding decay widths are denoted as $\Gamma_{p} \equiv \Gamma(\Lambda p \rightarrow n p)$ and $\Gamma_{n} \equiv \Gamma(\Lambda n \rightarrow n n)$, respectively. Just after the $\Lambda$-decays, the resultant nucleons are still within the nucleus and in their way out they can interact strongly with any nucleon of the nucleus. Finally, two or more nucleons are ejected from the nucleus and it is the nucleon spectra of these emitted nucleons the magnitude which can be measured. For review articles one can see [1,2], while some of the experimental works are [3-11].

For the non-mesonic $\Lambda$-decay, there are two quantities which deserves special attention. The first one is the ratio $\Gamma_{n / p} \equiv \Gamma_{n} / \Gamma_{p}$ where theory predicts a value smaller than 0.5 , while the so-called experimental result has a value closer to one. The second quantity is the asymmetry of the protons emitted in the NM decay of polarized hypernuclei. In this case, data indicate a value close to zero, while most of the theoretical works predict a large negative number. Just recently, the incorporation of the $\sigma$-meson suggests a solution to this problem (see [12,13], and references therein). In the present work, however, we will not deal with the asymmetry. For $\Gamma_{n / p}$, the connection between the theory and the experimental results is not straightforward because there is no direct measurement of this ratio. Both $\Gamma_{n}$ and $\Gamma_{p}$ are the decay widths of the sodesigned primaries disintegration and as mentioned above, data give us results on the nucleon spectra which emerge from the nucleus. The connection between $\Gamma_{n / p}$ and the nucleon spectra is a theoretical problem in itself. Before going on, it is interesting to resume some of the theoretical efforts in dealing with $\Gamma_{n / p}$.

The main ingredients required in the evaluation of the transition rate $(\Lambda N \rightarrow n N)$, are the transition potential and the wave functions which represent the $\Lambda$ and the nucleons. The first microscopic scheme for $\Gamma_{n / p}$ has been proposed by Adams [14], who has used the nuclear matter framework, one pion exchange model (OPE), $\Delta T=1 / 2$ piece of the $\Lambda N \pi$ coupling and short range correlations (SRC). While this model fairly reproduce the total NM decay width $\Gamma_{\mathrm{NM}}$, it produce ratios smaller than 0.20. Some of the improvements over Adams' model are: (i) the inclusion of heavier mesons than the pion in the $\Lambda N \rightarrow N N$-transition potential [15-24]; (ii) the inclusion of interaction terms that violates the isospin $\Delta T=1 / 2$ rule has been considered in [25,27]. Alternatively, (iii) the transition potential can be described in terms of the quark degree of freedom [28-32], which automatically introduces the $\Delta T=3 / 2$ contribution. And (iv) the employment of finite nucleus wave functions instead of plane waves, a scheme usually called wave function method (WFM) [16,17,19,20,22,23,26,27]. This list does not pretend to be complete. However, we should mention that in all these works the discrepancy between theory and experiment remains.

We turn now to the interpretation of data. As mentioned, it is the spectra of nucleons emerging from the nucleus, the quantity which is measured. The nucleons originated in the $\Lambda$-decay 
interact strongly with others nucleons before leaving the nucleus. These interactions are called final state interactions (FSI). Again, we can distinguish two issues: (i) the primary decays and (ii) a model for the FSI. Regarding the first point, we should note that $(\Lambda N \rightarrow n N)$ is not the only NM-weak decay mechanism: the NM-decay width can be also stimulated by two-nucleons $(\Lambda N N \rightarrow n N N)$. The corresponding decay width is called $\Gamma_{2}$. This two-body induced decay is originated from ground state correlation in the hypernuclei [33-38]. An estimation of $\Gamma_{2}$ is important in the analysis of data, because this process is a source of nucleons which cannot be distinguished from those stemming from $\Gamma_{1} \equiv \Gamma_{n}+\Gamma_{p}$. However, the two-nucleon induced decays are not included in the present calculation.

There are several models for the FSI among which we focus on two of them: the intranuclear cascade code (INC) [35,39-41] and the microscopic model developed in [42]. The INC is a semi-phenomenological approach. The starting point in the INC is the microscopic evaluation of $\Gamma_{n}, \Gamma_{p}$ and $\Gamma_{2}$. Afterwards, the nucleons produced in the weak decay are followed in a semi-classical manner until they leave the nucleus. By means of this emulation of the physical conditions of the hypernuclear decay, a more accurate agreement between the theoretical results and the data is achieved. The analysis of the experimental information using the INC, produce ratios $\left(\Gamma_{n / p}\right)^{\exp } \sim 0.4-0.6$. However, one limitation of the INC is that the quantum interference terms between the primary weak decay reaction $(\Lambda N \rightarrow N N)$ and any other reaction which has the same initial and final state, such as $(\Lambda N \rightarrow N N \rightarrow N N)$, cannot be included. On the other hand and to the best of our knowledge, the microscopic model described in [42], is the only microscopic model which puts in the same level of theoretical effort the weak decay mechanism and the FSI. Due to its character, the microscopic model automatically includes the quantum interference terms. Some details of the microscopic model are given in the next section.

While the theoretical prediction starts with the primary decay and should end in the nucleon spectra, the analysis of the experiment begins with the spectra, goes back and a value for $\Gamma_{n / p}$ is determined. This is done using several models, among which the INC is certainly one of the more elaborated ones. It should be noted, that the INC is employed in two ways: for the theoretical prediction of the nucleon spectra and for the interpretation of data. In any case, for the extraction of the $\Gamma_{n / p}$-experimental value one needs two theoretical inputs: $\Gamma_{2}$ and the FSI. Now, if several models for these inputs produce the same ratio $\Gamma_{n / p}$, it is reasonable to name it as the experimental value for $\Gamma_{n / p}$. Therefore, an accurate evaluation of the quantum interference terms together with an alternative formalism to the INC, is important.

In the present contribution, we further developed the microscopic model of [42] presenting results for the nucleon spectra, with emphasis in the quantum interference terms. The paper is organized as follows. In Section 2 we present the microscopic model for the nucleon spectra. This is done in general terms, including the two-body induced contribution. In Section 3, explicit expression within the first order contribution to the RPA are shown. In Section 4, the numerical results are discussed and finally, in Section 5 we give our conclusions.

\section{The microscopic model for the nucleon spectra}

In this section the microscopic model developed in [42] is briefly summarized with the addition of $\Gamma_{2}$. In fact, $\Gamma_{2}$ is the sum of three terms: $\Gamma_{n n} \equiv \Gamma(\Lambda n n \rightarrow n n n), \Gamma_{n p} \equiv \Gamma(\Lambda n p \rightarrow n n p)$ and $\Gamma_{p p} \equiv \Gamma(\Lambda p p \rightarrow n p p)$ (for details see [38]). The inclusion of $\Gamma_{2}$ in this section is done for completeness. As mentioned in the introduction, this quantity is not included in the numerical results. We are interested in reporting expressions for the single and double nucleon spectra: $N_{N}$ and $N_{N N^{\prime}}$, which represent the number of nucleons of kind $N=n, p$ vs. its kinetic energy, $T_{N}$ 
and number of pairs of nucleons $N N^{\prime}$ vs. $T_{N}+T_{N^{\prime}}$ or vs. the relative angle between $N$ and $N^{\prime}$, $\cos \left(\theta_{N N^{\prime}}\right)$, respectively. Without FSI, the expressions for $N_{N}$ and $N_{N N^{\prime}}$ are,

$$
\begin{aligned}
& N_{n}^{0}=2 \bar{\Gamma}_{n}+\bar{\Gamma}_{p}+3 \bar{\Gamma}_{n n}+2 \bar{\Gamma}_{n p}+\bar{\Gamma}_{p p}, \\
& N_{p}^{0}=\bar{\Gamma}_{p}+\bar{\Gamma}_{n p}+2 \bar{\Gamma}_{p p}, \\
& N_{n n}^{0}=\bar{\Gamma}_{n}+3 \bar{\Gamma}_{n n}+\bar{\Gamma}_{n p}, \\
& N_{n p}^{0}=\bar{\Gamma}_{p}+2 \bar{\Gamma}_{n p}+2 \bar{\Gamma}_{p p}, \\
& N_{p p}^{0}=\bar{\Gamma}_{p p}
\end{aligned}
$$

where we have used the normalization, $\bar{\Gamma}_{N} \equiv \Gamma_{N} / \Gamma_{\mathrm{NM}}$, with $\Gamma_{\mathrm{NM}}=\Gamma_{1}+\Gamma_{2}$. The index 0 , indicates that there is no FSI. The multiplicative factors in the right-hand side of these equations are the number of particles (pairs of particles) of kind $N\left(N N^{\prime}\right)$ produces by the primary decay. Explicit expressions for $\Gamma_{1}$ and $\Gamma_{2}$ are reported in [24] and [38], respectively. As mentioned, the nucleon spectra are the number of particles (or pairs of particles) which angle or kinetic energy lays within a certain range. Therefore, $\Gamma_{1}$ and $\Gamma_{2}$ are cut into pieces which correspond to certain angles- or kinetic energies-ranges. This is easily implemented by adding steps functions (in the integrand of $\Gamma_{1}$ and $\Gamma_{2}$ ), which limit the integration to such ranges.

The next step is the inclusion of the FSI. To this end, we introduce the quantity $\Gamma_{i, i^{\prime} \rightarrow j}$. This function results from evaluating any possible Goldstone diagram for the $\Lambda$-weak decay, where the strong interaction is present after the weak decay takes place. The index, $j$, is the final state (i.e., the emitted nucleons), taking the values: $j=n n, n p, n n n, n n p$, etc. At variance, the indices $i, i^{\prime}$ refer to the two primary weak decays of each diagram and can have the values $i$ (or $\left.i^{\prime}\right)=n, p, n n, n p, p p$; which stand for the transitions amplitudes $(\Lambda n \rightarrow n n),(\Lambda p \rightarrow n p)$, $(\Lambda n n \rightarrow n n n),(\Lambda n p \rightarrow n n p)$ and $(\Lambda p p \rightarrow n p p)$, respectively. It is important to be aware of the fact that the decay widths, $\Gamma_{n}, \Gamma_{p}, \Gamma_{n n}$, etc., are the square of a transition amplitude $((\Lambda N \rightarrow$ $n N)$ or $(\Lambda N N \rightarrow n N N))$. In this sense, $\Gamma_{i, i^{\prime} \rightarrow j}$ does not represent only a decay width, but also some interference terms, which are pure quantum mechanical effects.

By the addition of the FSI in Eqs. (2.1)-(2.5) we obtained,

$$
\begin{aligned}
& N_{n}=2 \bar{\Gamma}_{n}+\bar{\Gamma}_{p}+3 \bar{\Gamma}_{n n}+2 \bar{\Gamma}_{n p}+\bar{\Gamma}_{p p}+\sum_{i, i^{\prime} ; j} N_{j(n)} \bar{\Gamma}_{i, i^{\prime} \rightarrow j} \\
& N_{p}=\bar{\Gamma}_{p}+\bar{\Gamma}_{n p}+2 \bar{\Gamma}_{p p},+\sum_{i, i^{\prime} ; j} N_{j(p)} \bar{\Gamma}_{i, i^{\prime} \rightarrow j} \\
& N_{n n}=\bar{\Gamma}_{n}+3 \bar{\Gamma}_{n n}+\bar{\Gamma}_{n p}+\sum_{i, i^{\prime} ; j} N_{j(n n)} \bar{\Gamma}_{i, i^{\prime} \rightarrow j} \\
& N_{n p}=\bar{\Gamma}_{p}+2 \bar{\Gamma}_{n p}+2 \bar{\Gamma}_{p p}+\sum_{i, i^{\prime} ; j} N_{j(n p)} \bar{\Gamma}_{i, i^{\prime} \rightarrow j} \\
& N_{p p}=\bar{\Gamma}_{p p}+\sum_{i, i^{\prime} ; j} N_{j(p p)} \bar{\Gamma}_{i, i^{\prime} \rightarrow j}
\end{aligned}
$$

where the factors $N_{j(N)}$ are the numbers of nucleons of the type $N$ in the state $j$. In the same way, $N_{j\left(N N^{\prime}\right)}$ are the numbers of pairs of nucleons of the type $N N^{\prime}$ in the state $j$. Let us give two examples. If $j=n p, N_{n p(n)}=1, N_{n p(p)}=1, N_{n p(n n)}=0, N_{n p(n p)}=1, N_{n p(p p)}=0$; and if $j=$ $n n n, N_{n n n(n)}=3, N_{n n n(p)}=0, N_{n n n(n n)}=3, N_{n n n(n p)}=0, N_{n n n(p p)}=0$. In these expressions, the summation over $i, i^{\prime}$ and $j$, runs over the values of these indices mentioned above. 
$A_{1}$

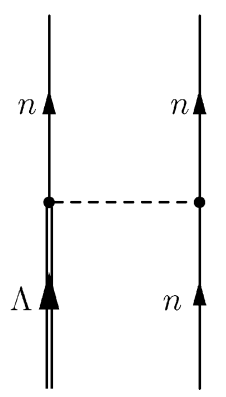

$A_{2}$

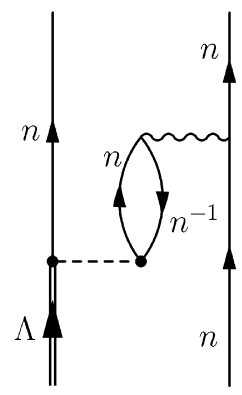

$A_{3}$

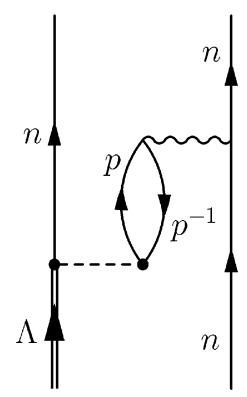

Fig. 1. Amplitudes $A_{1}, A_{2}$ and $A_{3}$, described in the text. A dashed line represents the $\Lambda N$-transition potential and the wavy line is the strong residual interaction.

Before we end this section, we make some further considerations about the quantum interference terms. All decay widths come from the square of a transition amplitude. When no FSI are included, the only transitions amplitudes are $(\Lambda N \rightarrow n N)$ and $(\Lambda N N \rightarrow n N N)$. For simplicity, we focus on $(\Lambda N \rightarrow n N)$. There is no interference term between $(\Lambda n \rightarrow n n)$ and $(\Lambda p \rightarrow n p)$ because the final state is different. When the FSI come into play, the strong interaction allows many others transitions amplitudes. In this case, as different reactions can end in the same final state and as the initial state is the same hypernuclear state for all the processes, the total transition amplitude is the sum of all these terms. The squares of the individual terms, are the decay widths, while the crossed products are the interference terms. Within our model, $\Gamma_{i, i^{\prime} \rightarrow j}$ contain both decay widths and interference terms. Note that the strong interaction is present in $\Gamma_{2}$ as a ground state correlation, that is, the strong interaction acts before the weak transition potential.

As an additional comment, we note that the interference terms can be grouped into two categories: (a) the ones with $i=i^{\prime}$ and (b) the ones with $i \neq i^{\prime}$. It is perhaps more convenient to explain these categories by means of an example. Let us propose three transition amplitudes: $A_{1}$, $A_{2}$ and $A_{3}$ shown in Fig. 1 . The $A_{1}$-transition amplitude represents the $\Lambda n \rightarrow n n$-primary decay, while $A_{2}$ and $A_{3}$ include secondary decays, due to the action of the strong interaction. There is an interference term between $A_{1}$ and $A_{2}\left(i=i^{\prime}\right)$, where the strong interaction appears in first order. It is clear that the square of $A_{1}+A_{2}$ is positive, but the interference terms between both terms can be either positive or negative. The interference terms between $A_{1}$ and $A_{3}$ is an example of the $i \neq i^{\prime}$-category. It is convenient to discuss these two interference contributions in terms of self-energy diagrams. For simplicity, we refer to the $d d d$-diagram in Fig. 4 , in the next section. This diagram has two one particle-one hole bubbles. When all particles (holes) are neutron particles (neutron holes) we have the interference term between $A_{1}$ and $A_{2}$. When one particle-hole bubble is an $n n^{-1}$, the other one is a $p p^{-1}$ and the cut (which represents the physical state) is in the $n n^{-1}$-bubble, we have the interference terms between $A_{1}$ and $A_{3}$.

In order to avoid misinterpretations, two final points should be addressed. In first place, the index $i$ (or $i^{\prime}$ ) refers always to the primary decay, where the primary decay is the one induced by the $\Lambda N$-transition potential. In Fig. $1, A_{2}$ has $i=n$, and for $A_{3}$ we have $i=p$. The second point deals with the $\Lambda N$-transition potential. Also from the $A_{2}$ and $A_{3}$-amplitudes, it is appealing to re-define the transition potential as a dressed one, with the inclusion of particle-hole polarization effects. Such a potential, is still a two-body operator and in this case, the definition of $i$ (in $A_{3}$, for example) would be ambiguous. The fallacy in the idea of using this dressed transition potential 


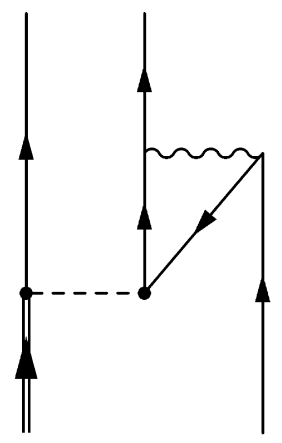

Fig. 2. Example of an $A_{2}$-exchange term.

lies in the following: the complete expression for $A_{2}$ (or $A_{3}$ ) has also three exchange terms. One of them is shown in Fig. 2. From this figure, it is clear that the combined action of the $\Lambda N$ transition potential and the nuclear residual interaction cannot be replaced by a single two-body operator.

\section{Explicit expressions for $\Gamma_{i, i^{\prime} \rightarrow j}$}

In this section we present expressions for the functions $\Gamma_{i, i^{\prime} \rightarrow j}$, using non-relativistic nuclear matter together with the local density approximation (LDA) [43], which allows us to discuss any particular hypernucleus. Eqs. (2.6)-(2.10) are general expressions for $N_{N}$ and $N_{N N^{\prime}}$. Although $\Gamma_{i, i^{\prime} \rightarrow j}$ is completely defined, one has to choose some set of Goldstone diagrams which represent the $\Lambda$-decay to obtain the explicit expressions for this function. This set has an infinite number of diagrams and a priori any sub-set of diagrams could be equally important. In this work and as a first step in the evaluation of the nucleon spectra, we have decided to study the RPA-like diagrams. The direct part of the RPA, known as ring approximation, is perhaps the simplest manner to implement the strong interaction into this problem. The Pauli exchange terms are known to be important for these particular set of diagrams (see [44]) and consequently the RPA looks as a natural first step. In [42] we have presented expressions for $\Gamma_{i, i^{\prime} \rightarrow j}$ within the ring approximation. However, no comparison with data has been done, because of the absence of the Pauli exclusion principle.

Let us call by $\Gamma$ a generic function which can be either $\Gamma_{1}, \Gamma_{2}$ or $\Gamma_{i, i^{\prime} \rightarrow j}$. Instead of giving the expression for $\Gamma$, it is more convenient to work with the partial decay width $\Gamma\left(k, k_{F_{n}}, k_{F_{p}}\right)$, where, $k$ is the $\Lambda$ energy-momentum, $k_{F_{n}}$ and $k_{F_{p}}$ are the Fermi momentum for neutrons and protons, respectively. To evaluate $\Gamma(k)$ for a particular nucleus one can use either an effective Fermi momentum or the local density approximation. In this work the LDA is adopted, which make $k_{F_{n}}$ and $k_{F_{p}}$ position-dependent. They are defined as $k_{F_{n(p)}}(r)=\hbar c\left(3 \pi^{2} \rho_{n(p)}(r)\right)^{1 / 3}$, where $\rho_{n}(r)=\rho(r) N /(N+Z)$ and $\rho_{p}(r)=\rho(r) Z /(N+Z)$, with $\rho(r), N$ and $Z$ being, respectively, the density profile, number of neutrons and number of protons of the nuclear core of the hypernucleus. In the last case, it is equivalent to write the function $\Gamma\left(k, k_{F_{n}}, k_{F_{p}}\right)$ in terms of the densities as $\Gamma\left(k, \rho_{n}(r), \rho_{p}(r)\right)$. The LDA reads,

$$
\Gamma(k)=\int d \boldsymbol{r} \Gamma\left(k, \rho_{n}(r), \rho_{p}(r)\right)\left|\psi_{\Lambda}(\boldsymbol{r})\right|^{2},
$$



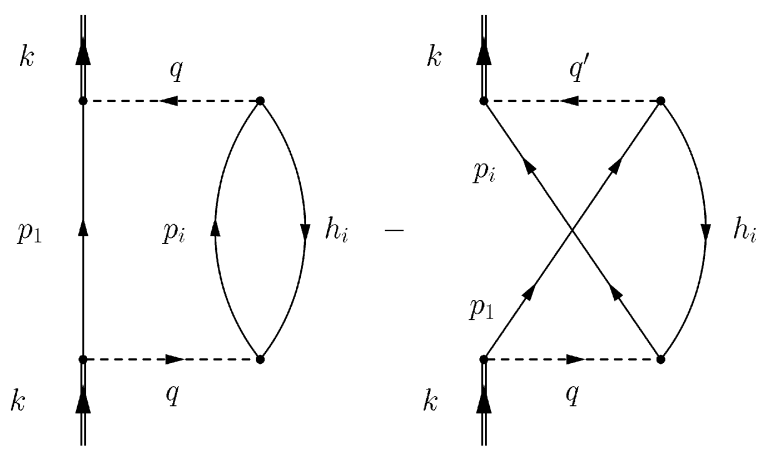

Fig. 3. Goldstone diagrams for the direct and exchange contributions to the $\Lambda$ decay width, respectively.

where for the $\Lambda$ wave function $\psi_{\Lambda}(\boldsymbol{r})$, we take the $1 s_{1 / 2}$ wave function of a harmonic oscillator. The final result is obtained by averaging over the $\Lambda$ momentum distribution, $\left|\tilde{\psi}_{\Lambda}(\boldsymbol{k})\right|^{2}$, as follows,

$$
\Gamma=\int d \boldsymbol{k} \Gamma(k)\left|\tilde{\psi}_{\Lambda}(\boldsymbol{k})\right|^{2},
$$

where $\tilde{\psi}_{\Lambda}(\boldsymbol{k})$ is the Fourier transform of $\psi_{\Lambda}(\boldsymbol{r})$ and $k_{0}=E_{\Lambda}(\boldsymbol{k})+V_{\Lambda}$, being $V_{\Lambda}$ the binding energy for the $\Lambda$.

As remarked in the last section, the expressions for $\Gamma_{i, i^{\prime} \rightarrow j}$ are obtained as if they were decay widths. In this spirit, it is convenient to overview the derivation of $\Gamma_{1}$, because this simplifies the derivation of $\Gamma_{i, i^{\prime} \rightarrow j}$. In Fig. 3 we show the direct and exchange $\Gamma_{1}$-contributions. The distinction between $\Gamma_{n}$ and $\Gamma_{p}$ can be ascribed to the isospin of the hole line, where when $h_{i}$ is a neutron(proton-)hole we are considering the $\Gamma_{n}\left(\Gamma_{p}\right)$-decay width. To get the analytical expressions for these diagrams we have employed the Golstone rules (for a more detailed version, see [24]),

$$
\begin{aligned}
\Gamma_{t_{h i}}^{\text {ant }}\left(k, k_{F_{n}}, k_{F_{p}}\right)= & -2 \operatorname{Im} \int \frac{d^{4} p_{1}}{(2 \pi)^{4}} \int \frac{d^{4} p_{i}}{(2 \pi)^{4}} G_{\text {part }}\left(p_{1}\right) G_{\text {part }}\left(p_{i}\right) G_{\text {hole }}\left(h_{i}\right) \\
& \times \frac{1}{4} \sum_{\text {all spins }, t_{p 1}, t_{p i}}\left\langle\gamma_{\Lambda}\left|\left(V^{\Lambda N}\right)^{\dagger}\right| \gamma_{p 1} \gamma_{p i} \gamma_{h i}\right\rangle_{\text {ant }}\left\langle\gamma_{p 1} \gamma_{p i} \gamma_{h i}\left|V^{\Lambda N}\right| \gamma_{\Lambda}\right\rangle_{\text {ant }},
\end{aligned}
$$

where for simplicity, $\gamma_{i}$ represents the spin $(s)$, isospin $(t)$ and energy-momentum of the particle $i$. The energy-momentum carried by the transition potential, $V^{\Lambda N}$, cannot be specified until we separate the direct and the exchange terms. The meaning of the subindexes $p i$ and $h i$ is shown in Fig. 3, where due to the momentum conservation, $\boldsymbol{h}_{i}=\boldsymbol{p}_{1}+\boldsymbol{p}_{i}-\boldsymbol{k}$. The direct and exchange matrix elements are,

$$
\begin{aligned}
& \left\langle\gamma_{\Lambda}\left|\left(V^{\Lambda N}\right)^{\dagger}\right| \gamma_{p 1} \gamma_{p i} \gamma_{h i}\right\rangle_{\text {ant }}\left\langle\gamma_{p 1} \gamma_{p i} \gamma_{h i}\left|V^{\Lambda N}\right| \gamma_{\Lambda}\right\rangle_{\text {ant }} \\
& =\left\langle\gamma_{\Lambda}\left|\left(V^{\Lambda N}(q)\right)^{\dagger}\right| \gamma_{p 1} \gamma_{p i} \gamma_{h i}\right\rangle\left\langle\gamma_{p 1} \gamma_{p i} \gamma_{h i}\left|V^{\Lambda N}(q)\right| \gamma_{\Lambda}\right\rangle \\
& \quad-\left\langle\gamma_{\Lambda}\left|\left(V^{\Lambda N}\left(q^{\prime}\right)\right)^{\dagger}\right| \gamma_{p i} \gamma_{p 1} \gamma_{h i}\right\rangle\left\langle\gamma_{p 1} \gamma_{p i} \gamma_{h i}\left|V^{\Lambda N}(q)\right| \gamma_{\Lambda}\right\rangle,
\end{aligned}
$$

where the minus sign comes from the crossing of the fermionic lines. Due to energy-momentum conservation, we have $q=k-p_{1}$ and $q^{\prime}=k-p_{i}$. The first (second) term in the right-hand side of Eq. (3.4) originates the direct (exchange) contribution to the decay width. 
The particle and hole propagators are,

$$
G_{\text {part }}(p)=\frac{\theta\left(|\boldsymbol{p}|-k_{F}\right)}{p_{0}-E_{N}(\boldsymbol{p})-V_{N}+i \varepsilon}
$$

and

$$
G_{\text {hole }}(h)=\frac{\theta\left(k_{F}-|\boldsymbol{h}|\right)}{h_{0}-E_{N}(\boldsymbol{h})-V_{N}-i \varepsilon},
$$

where $E_{N}$ is the nucleon total free energy and $V_{N}$ is the nucleon binding energy. The value of the Fermi momentum depends on whether we have a proton or a neutron. The $\Lambda N \rightarrow N N$ transition potential $V^{\Lambda N}$, is,

$$
V^{\Lambda N(N N)}(q)=\sum_{\tau_{\Lambda(N)}=0,1} \mathcal{O}_{\tau_{\Lambda(N)}} \mathcal{V}_{\tau_{\Lambda(N)}^{\Lambda N(N N)}}^{\Lambda}(q),
$$

where we have included the nuclear residual interaction $V^{N N}$, which is employed soon. The isospin dependence is given by,

$$
\mathcal{O}_{\tau_{\Lambda(N)}}= \begin{cases}1, & \text { for } \tau_{\Lambda(N)}=0, \\ \boldsymbol{\tau}_{1} \cdot \boldsymbol{\tau}_{2}, & \text { for } \tau_{\Lambda(N)}=1 .\end{cases}
$$

The values $\tau=0,1$ stand for the isoscalar and isovector parts of the interaction, respectively. The spin and momentum dependence of the transition potential is,

$$
\begin{aligned}
\mathcal{V}_{\tau_{\Lambda}}^{\Lambda N}(q)= & \left(G_{F} m_{\pi}^{2}\right)\left\{S_{\tau_{\Lambda}}(q) \boldsymbol{\sigma}_{1} \cdot \hat{\boldsymbol{q}}+S_{\tau_{\Lambda}}^{\prime}(q) \boldsymbol{\sigma}_{2} \cdot \hat{\boldsymbol{q}}+P_{L, \tau_{\Lambda}}(q) \boldsymbol{\sigma}_{1} \cdot \hat{\boldsymbol{q}} \boldsymbol{\sigma}_{2} \cdot \hat{\boldsymbol{q}}+P_{C, \tau_{\Lambda}}(q)\right. \\
& \left.+P_{T, \tau_{\Lambda}}(q)\left(\sigma_{1} \times \hat{\boldsymbol{q}}\right) \cdot\left(\boldsymbol{\sigma}_{2} \times \hat{\boldsymbol{q}}\right)+i S_{V, \tau_{\Lambda}}(q)\left(\sigma_{1} \times \boldsymbol{\sigma}_{2}\right) \cdot \hat{\boldsymbol{q}}\right\},
\end{aligned}
$$

where the quantities $S_{\tau_{\Lambda}}(q), S_{\tau_{\Lambda}}^{\prime}(q), P_{L, \tau_{\Lambda}}(q), P_{C, \tau_{\Lambda}}(q), P_{T, \tau_{\Lambda}}(q)$ and $S_{V, \tau_{\Lambda}}(q)$ contain short range correlations (SRC) and are given in Appendix B of [24]. They are built up from the full one-meson-exchange potential (OMEP), which involves the complete pseudoscalar and vector meson octets $\left(\pi, \eta, K, \rho, \omega, K^{*}\right)$. It is self evident that the $S$ - $(P)$-terms are the parity violating (parity conserving) terms of the transition potential.

The nuclear residual interaction is drawn as,

$$
\mathcal{V}_{\tau_{N}}^{N N}(q)=\left(\frac{f_{\pi}^{2}}{m_{\pi}^{2}}\right)\left\{\mathcal{V}_{C, \tau_{N}}(q)+\mathcal{V}_{\sigma, \tau_{N}}(q) \boldsymbol{\sigma}_{1} \cdot \boldsymbol{\sigma}_{2}+\mathcal{V}_{L, \tau_{N}}(q) \boldsymbol{\sigma}_{1} \cdot \hat{\boldsymbol{q}} \boldsymbol{\sigma}_{2} \cdot \hat{\boldsymbol{q}}\right\},
$$

where the functions $\mathcal{V}_{C, \tau_{N}}(q), \mathcal{V}_{\sigma, \tau_{N}}(q)$ and $\mathcal{V}_{L, \tau_{N}}(q)$ are adjusted to reproduce any effective OMEP-nuclear residual interaction.

We have now all the elements required for the evaluation of $\Gamma_{i, i^{\prime} \rightarrow j}$ within the RPA approximation. The ring approximation has been discussed in [42], where the advantage of the ring series is that it can be summed up to infinite order in a very simple way. The situation is different in the RPA, where the corresponding series can be summed up only in some particular cases: when the nuclear residual interaction is represented by a contact or by a separable interaction. For a general finite range interaction there is no way out but to evaluate each exchange term individually. The problem is quite involved because matrix elements must be antisymmetrized for both $V^{\Lambda N}$ and $V^{N N}$. The lowest order RPA-contribution is the one in which $V^{N N}$ appears in first order, being the only one reported in the present contribution. In the lowest RPA-contribution, there are two matrix elements with $V^{\Lambda N}$ and one with $V^{N N}$. Each matrix element has a direct and an exchange part. This makes a total of eight different diagrams, which are shown in Fig. 4. Unfortunately, in nuclear matter each diagram must be evaluated individually. In the next section and by means of 
$d d d$

dde

edd

ded
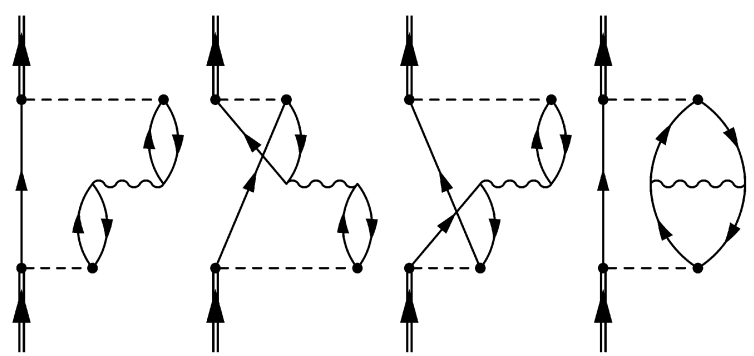

dee

eed

ede

eee
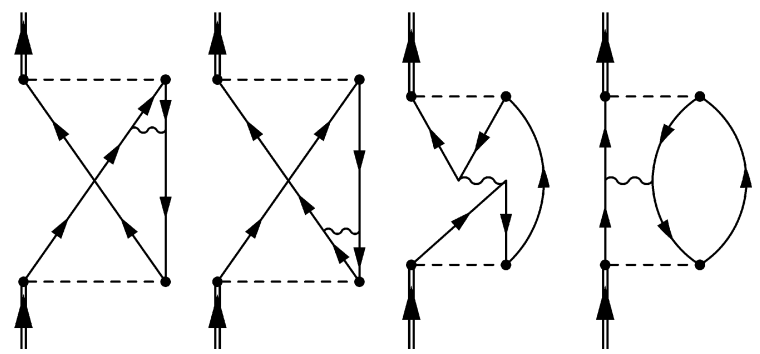

Fig. 4. Goldstone diagrams for the first order contribution to the RPA-series. The meaning of the notation is explained in the text. The dashed and wavy lines stand for $V^{\Lambda N}$ and $V^{N N}$, respectively. An up (down) going arrow represents a particle (hole), while an arrow with a wide line represents the $\Lambda$.

the numerical analysis, we discuss this approximation. Using the standard Goldstone rules, this lowest order RPA-contribution is written as,

$$
\begin{aligned}
\Gamma_{i, i^{\prime} \rightarrow} & j\left(k, k_{F_{n}}, k_{F_{p}}\right) \\
= & -2 \operatorname{Im} \int \frac{d^{4} p_{1}}{(2 \pi)^{4}} \int \frac{d^{4} h_{i}}{(2 \pi)^{4}} \int \frac{d^{4} h_{i^{\prime}}}{(2 \pi)^{4}} \frac{1}{4} \sum G_{\text {part }}\left(p_{1}\right) G_{\text {part }}\left(p_{i}\right) \\
& \times G_{\text {part }}\left(p_{i^{\prime}}\right) G_{\text {hole }}\left(h_{i}\right) G_{\text {hole }}\left(h_{i^{\prime}}\right) \\
& \times\left\langle\gamma_{\Lambda}\left|\left(V^{\Lambda N}\left(q^{\prime}\right)\right)^{\dagger}\right| \gamma_{p 1} \gamma_{p i^{\prime}} \gamma_{h i^{\prime}}\right\rangle_{\text {ant }}\left\langle\gamma_{p 1} \gamma_{p i^{\prime}} \gamma_{h i^{\prime}}\left|V^{N N}(t)\right| \gamma_{p 1} \gamma_{p i} \gamma_{h i}\right\rangle_{\text {ant }} \\
& \times\left\langle\gamma_{p 1} \gamma_{p i} \gamma_{h i}\left|V^{\Lambda N}(q)\right| \gamma_{\Lambda}\right\rangle_{\text {ant }} .
\end{aligned}
$$

The summation runs over all spins, while the isospin sum cannot be specified until one sets the final state $j$. The energy-momentum carried by each fermionic line is shown in Fig. 5 for the direct term. Due to the energy-momentum conservation in each vertex, for all the contributions we have $p_{i}=h_{i}+k-p_{1}$ and $p_{i^{\prime}}=h_{i^{\prime}}+k-p_{1}$; while the energy-momentum carried by $V^{N N}$ and $V^{\Lambda N}$ depends on the topology of each diagram. These values, together with some more details on the exchange terms are specified in Appendix A.

It is convenient to re-write Eq. (3.11) as,

$$
\Gamma_{i, i^{\prime} \rightarrow j}=\sum_{\alpha, \beta, \delta=d, e} \Gamma_{i, i^{\prime} \rightarrow j}^{\alpha \beta \delta},
$$




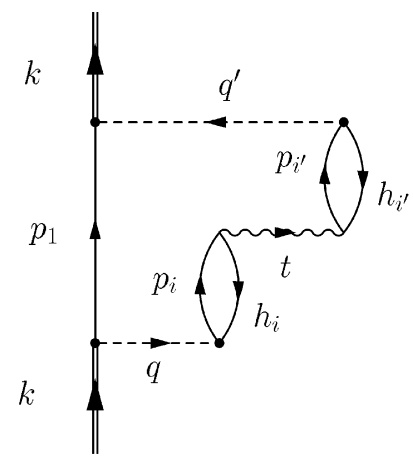

Fig. 5. Here we have repeated the $d d d$-contribution from Fig. 4, in order to assign values to the momentum transfer. The remainder contributions have the same pattern.

where the super-indexes $\alpha, \beta$ and $\delta$, refer to the direct $(d)$ and exchange $(e)$ matrix elements $\left\langle\left|\left(V^{\Lambda N}\right)^{\dagger}\right|\right\rangle_{d+e},\left\langle\left|V^{N N}\right|\right\rangle_{d+e}$ and $\left\langle\left|V^{\Lambda N}\right|\right\rangle_{d+e}$, respectively. For instance, for the direct contribution, we have $\alpha \beta \delta=d d d$. Note that $\alpha$ ( $\beta$ and $\delta)$, can take the 'value' $d$ or $e$, which makes the total of eight different contributions, being $d d d$ the first order term to the ring series.

By inspection of the diagrams in Fig. 4, we notice that the strong interaction split these graphs into two pieces. In the lower piece particles are named as $p_{1}$ and $p_{i}$, while in the upper portion we have $p_{1}$ and $p_{i^{\prime}}$. Each diagram gives two contributions: the first one with $p_{1}$ and $p_{i}$ ( $p_{1}$ and $p_{i^{\prime}}$ ) on (off) the mass shell, and the inverse situation. Then, it is convenient to differentiate these two possibilities by the super-index $l$ and $u$, respectively, as follows,

$$
\begin{aligned}
& \left.\Gamma_{i, i^{\prime} \rightarrow j}^{\alpha \beta \delta, u}\left(k, k_{F_{n}}, k_{F_{p}}\right) \equiv \Gamma_{i, i^{\prime} \rightarrow j}^{\alpha \beta \delta}\left(k, k_{F_{n}}, k_{F_{p}}\right)\right|_{p 1, p i^{\prime} \text { on the mass shell }}, \\
& \left.\Gamma_{i, i^{\prime} \rightarrow j}^{\alpha \beta \delta, l}\left(k, k_{F_{n}}, k_{F_{p}}\right) \equiv \Gamma_{i, i^{\prime} \rightarrow j}^{\alpha \beta \delta}\left(k, k_{F_{n}}, k_{F_{p}}\right)\right|_{p 1, p i \text { on the mass shell }}
\end{aligned}
$$

The origin of these two terms comes from the energy integration in Eq. (3.11), which produces an expression with two poles: one in the upper and the other in the lower part of the diagram. Clearly, the distinction between $l$ and $u$, is redundant for the direct contribution, due to the symmetry of the diagram.

In order to perform the summation on spin and isospin quantum numbers it is convenient to write,

$$
\Gamma_{i, i^{\prime} \rightarrow j}^{\alpha \beta \delta, l(u)}\left(k, k_{F_{n}}, k_{F_{p}}\right)=\sum_{\tau, \tau_{N}, \tau^{\prime}=0,1} \mathcal{T}_{i, i^{\prime} \rightarrow j ; \tau^{\prime} \tau_{N} \tau}^{\alpha \beta \delta, l(u)} \tilde{\Gamma}_{i, i^{\prime} \rightarrow j ; \tau^{\prime} \tau_{N} \tau}^{\alpha \beta \delta, l(u)}\left(k, k_{F_{n}}, k_{F_{p}}\right),
$$

where

$$
\begin{aligned}
& \mathcal{T}_{i, i^{\prime} \rightarrow j ; \tau^{\prime} \tau_{N} \tau}^{\alpha \beta \delta, u}=\sum_{u}\left\langle t_{\Lambda}\left|\mathcal{O}_{\tau^{\prime}}\right| t_{p 1} t_{p i^{\prime}} t_{h i^{\prime}}\right\rangle_{\alpha}\left\langle t_{p i^{\prime}} t_{h i^{\prime}}\left|\mathcal{O}_{\tau_{N}}\right| t_{p i} t_{h i}\right\rangle_{\beta}\left\langle t_{p 1} t_{p i} t_{h i}\left|\mathcal{O}_{\tau}\right| t_{\Lambda}\right\rangle_{\delta}, \\
& \mathcal{T}_{i, i^{\prime} \rightarrow j ; \tau^{\prime} \tau_{N} \tau}^{\alpha \beta \delta, l}=\sum_{l}\left\langle t_{\Lambda}\left|\mathcal{O}_{\tau^{\prime}}\right| t_{p 1} t_{p i^{\prime}} t_{h i^{\prime}}\right\rangle_{\alpha}\left\langle t_{p i^{\prime}} t_{h i^{\prime}}\left|\mathcal{O}_{\tau_{N}}\right| t_{p i} t_{h i}\right\rangle_{\beta}\left\langle t_{p 1} t_{p i} t_{h i}\left|\mathcal{O}_{\tau}\right| t_{\Lambda}\right\rangle_{\delta} .
\end{aligned}
$$

In the isospin summation, $l(u)$ means that the final state $j$ is in the lower (upper) piece of the diagram. To avoid confusion, we give an example: let $i=n, i^{\prime}=p$ and $j=n p$. The $l$ sum is zero because for the final state $j$, there are no protons in the lower portion of the diagram. The $u$ sum reduces to one term which isospin projections for each particles are $t_{p 1}=t_{p i}=t_{h i^{\prime}}=-1 / 2$ and $t_{p i^{\prime}}=t_{h i}=1 / 2$. 
Performing the energy integration, the spin summation and after some algebra, we obtain,

$$
\begin{aligned}
\tilde{\Gamma}_{i, i^{\prime} \rightarrow j ; \tau^{\prime} \tau_{N} \tau}^{\alpha \beta \delta, l}= & \left(G_{F} m_{\pi}^{2}\right)^{2} \frac{(-1)^{N_{F}}}{(2 \pi)^{7}}\left(\frac{f_{\pi}^{2}}{m_{\pi}^{2}}\right) \iiint d \boldsymbol{p}_{1} d \boldsymbol{h}_{i} d \boldsymbol{h}_{i^{\prime}} \mathcal{S}_{\tau^{\prime} \tau_{N} \tau}^{\alpha \beta \delta}\left(q, q^{\prime}, t\right) \theta\left(q_{0}\right) \theta\left(q_{0}^{\prime}\right) \\
& \times \theta\left(\left|\boldsymbol{p}_{1}\right|-k_{F p 1}\right) \theta\left(\left|\boldsymbol{p}_{i}\right|-k_{F p i}\right) \theta\left(k_{F h i}-\left|\boldsymbol{h}_{i}\right|\right) \\
& \times \theta\left(\left|\boldsymbol{p}_{i^{\prime}}\right|-k_{F p i^{\prime}}\right) \theta\left(k_{F h i^{\prime}}-\left|\boldsymbol{h}_{i^{\prime}}\right|\right) \\
& \times \frac{\mathcal{P}}{q_{0}^{\prime}-\left(E_{N}\left(\boldsymbol{p}_{i^{\prime}}\right)-E_{N}\left(\boldsymbol{h}_{i^{\prime}}\right)\right)} \delta\left(q_{0}-\left(E_{N}\left(\boldsymbol{p}_{i}\right)-E_{N}\left(\boldsymbol{h}_{i}\right)\right)\right) \\
\tilde{\Gamma}_{i, i^{\prime} \rightarrow j ; \tau^{\prime} \tau_{N} \tau}^{\alpha \beta \delta, u} & -\left(G_{F} m_{\pi}^{2}\right)^{2} \frac{(-1)^{N_{F}}}{(2 \pi)^{7}}\left(\frac{f_{\pi}^{2}}{m_{\pi}^{2}}\right) \iiint d \boldsymbol{p}_{1} d \boldsymbol{h}_{i} d \boldsymbol{h}_{i^{\prime}} \mathcal{S}_{\tau^{\prime} \tau_{N} \tau}^{\alpha \beta \delta}\left(q, q^{\prime}, t\right) \theta\left(q_{0}\right) \theta\left(q_{0}^{\prime}\right) \\
& \times \theta\left(\left|\boldsymbol{p}_{1}\right|-k_{F p 1}\right) \theta\left(\left|\boldsymbol{p}_{i}\right|-k_{F p i}\right) \theta\left(k_{F h i}-\left|\boldsymbol{h}_{i}\right|\right) \\
& \times \theta\left(\left|\boldsymbol{p}_{i^{\prime}}\right|-k_{F p i^{\prime}}\right) \theta\left(k_{F h i^{\prime}}-\left|\boldsymbol{h}_{i^{\prime}}\right|\right) \\
& \times \frac{\mathcal{P}}{q_{0}-\left(E_{N}\left(\boldsymbol{p}_{i}\right)-E_{N}\left(\boldsymbol{h}_{i}\right)\right)} \delta\left(q_{0}^{\prime}-\left(E_{N}\left(\boldsymbol{p}_{i^{\prime}}\right)-E_{N}\left(\boldsymbol{h}_{i^{\prime}}\right)\right)\right)
\end{aligned}
$$

In these expressions, $N_{F}$ is the number of crossing of fermionic lines and $\mathcal{P}$ indicates the principal value. Explicit expressions for the functions $\mathcal{S}_{\tau^{\prime} \tau_{N} \tau}^{\alpha \beta \delta}\left(q, q^{\prime}, t\right)$ can be found in Appendix A. Here $q\left(q^{\prime}\right)$ is the energy-momentum carried by the transition potential in the lower (upper) part of the diagram, while $t$ corresponds to the nuclear interaction. We present in this section explicit expressions for the direct contribution, whereas the exchange ones are shown in Appendix A. The $\mathcal{S}_{\tau^{\prime} \tau_{N} \tau}^{d d d}\left(q, q^{\prime}, t\right)$ reads,

$$
\begin{aligned}
\mathcal{S}_{\tau^{\prime} \tau_{N} \tau}^{d d d}(q)= & 4\left\{\left(S_{\tau^{\prime}}^{\prime} S_{\tau}^{\prime}+P_{C, \tau^{\prime}} P_{C, \tau}\right) \mathcal{V}_{C, \tau_{N}}+\left(S_{\tau^{\prime}} S_{\tau}+P_{L, \tau^{\prime}} P_{L, \tau}\right) \mathcal{V}_{L, \tau_{N}}\right. \\
& \left.+2\left(S_{V, \tau^{\prime}} S_{V, \tau}+P_{T, \tau^{\prime}} P_{T, \tau}\right) \mathcal{V}_{T, \tau_{N}}\right\},
\end{aligned}
$$

where for the direct contribution we have, $q^{\prime}=t=q$. By means of Eqs. (3.1) and (3.2), the dependence on the Fermi and $\Lambda$-momenta in the partial widths is eliminated. For the $(d d d)$ diagrams, we have $\tilde{\Gamma}_{i, i^{\prime} \rightarrow j ; \tau^{\prime} \tau_{N} \tau}^{d d d, l}=\tilde{\Gamma}_{i, i^{\prime} \rightarrow j ; \tau^{\prime} \tau_{N} \tau}^{d d d,} \equiv \tilde{\Gamma}_{i, i^{\prime} \rightarrow j ; \tau^{\prime} \tau_{N} \tau}^{d d} / 2$. By performing the summation over isospin for all possible primary decays and final states, we have,

$$
\begin{aligned}
\Gamma_{n, n \rightarrow n n}^{d d d}= & \tilde{\Gamma}_{n, n \rightarrow n n, 111}^{d d d, n}+\tilde{\Gamma}_{n, n \rightarrow n n, 000}^{d d d, n}+\tilde{\Gamma}_{n, n \rightarrow n n, 110}^{d d d, n}+\tilde{\Gamma}_{n, n \rightarrow n n, 101}^{d d d, n}+\tilde{\Gamma}_{n, n \rightarrow n n, 011}^{d d d, n} \\
& +\tilde{\Gamma}_{n, n \rightarrow n n, 100}^{d d d, n}+\tilde{\Gamma}_{n, n \rightarrow n n, 010}^{d d d, n}+\tilde{\Gamma}_{n, n \rightarrow n n, 001}^{d d d, n}, \\
\Gamma_{n, p \rightarrow n n}^{d d d}= & \tilde{\Gamma}_{n, p \rightarrow n n, 111}^{d d d, n}+\tilde{\Gamma}_{n, p \rightarrow n n, 000}^{d d d, n}+\tilde{\Gamma}_{n, p \rightarrow n n, 110}^{d d d, n}-\tilde{\Gamma}_{n, p \rightarrow n n, 101}^{d d d, n}-\tilde{\Gamma}_{n, p \rightarrow n n, 011}^{d d d, n} \\
& -\tilde{\Gamma}_{n, p \rightarrow n n, 100}^{d d d, n}-\tilde{\Gamma}_{n, p \rightarrow n n, 010}^{d d d, n}+\tilde{\Gamma}_{n, p \rightarrow n n, 001}^{d d d, n}, \\
\Gamma_{p, n \rightarrow n n}^{d d d}= & \tilde{\Gamma}_{p, n \rightarrow n n, 111}^{d d d, n}+\tilde{\Gamma}_{p, n \rightarrow n n, 000}^{d d d, n}-\tilde{\Gamma}_{p, n \rightarrow n n, 110}^{d d d, n}-\tilde{\Gamma}_{p, n \rightarrow n n, 101}^{d d d, n}+\tilde{\Gamma}_{p, n \rightarrow n n, 011}^{d d d, n} \\
& +\tilde{\Gamma}_{p, n \rightarrow n n, 100}^{d d d, n}-\tilde{\Gamma}_{p, n \rightarrow n n, 010}^{d d d, n}-\tilde{\Gamma}_{p, n \rightarrow n n, 001}^{d d d, n}, \\
\Gamma_{n, p \rightarrow n p}^{d d d}= & \tilde{\Gamma}_{n, p \rightarrow n p, 111}^{d d d, n}+\tilde{\Gamma}_{n, p \rightarrow n p, 000}^{d d d, n}+\tilde{\Gamma}_{n, p \rightarrow n p, 110}^{d d d, n}-\tilde{\Gamma}_{n, p \rightarrow n p, 101}^{d d d, n}-\tilde{\Gamma}_{n, p \rightarrow n p, 011}^{d d d, n} \\
& -\tilde{\Gamma}_{n, p \rightarrow n p, 100}^{d d d, n}-\tilde{\Gamma}_{n, p \rightarrow n p, 010}^{d d d, n}+\tilde{\Gamma}_{n, p \rightarrow n p, 001}^{d d d, n}, \\
\Gamma_{p, n \rightarrow n p}^{d d d}= & \tilde{\Gamma}_{p, n \rightarrow n p, 111}^{d d d, n}+\tilde{\Gamma}_{p, n \rightarrow n p, 000}^{d d d, n}-\tilde{\Gamma}_{p, n \rightarrow n p, 110}^{d d d, n}-\tilde{\Gamma}_{p, n \rightarrow n p, 101}^{d d d, n}+\tilde{\Gamma}_{p, n \rightarrow n p, 011}^{d d d, n} \\
& +\tilde{\Gamma}_{p, n \rightarrow n p, 100}^{d d d, n}-\tilde{\Gamma}_{p, n \rightarrow n p, 010}^{d d d, n}-\tilde{\Gamma}_{p, n \rightarrow n p, 001}^{d d d, n},
\end{aligned}
$$




$$
\begin{aligned}
\Gamma_{p, p \rightarrow n p}^{d d d}= & 4 \tilde{\Gamma}_{p, p \rightarrow n p, 111}^{d d d, p}+\tilde{\Gamma}_{p, p \rightarrow n p, 111}^{d d d, n}+\tilde{\Gamma}_{p, p \rightarrow n p, 000}^{d d d, n}-\tilde{\Gamma}_{p, p \rightarrow n p, 110}^{d d d, n}+\tilde{\Gamma}_{p, p \rightarrow n p, 101}^{d d d, n} \\
& -\tilde{\Gamma}_{p, p \rightarrow n p, 011}^{d d d, n}-\tilde{\Gamma}_{p, p \rightarrow n p, 100}^{d d d, n}+\tilde{\Gamma}_{p, p \rightarrow n p, 010}^{d d d, n}-\tilde{\Gamma}_{p, p \rightarrow n p, 001}^{d d d, n},
\end{aligned}
$$

where in the right-hand side of these equations, we have put an additional super-index ( $n$ or $p$ ) which is the isospin projection of the $p_{1}$ particle. As we are using different Fermi momenta for protons and neutrons, the knowledge of the isospin projection of each particle is required. This information about $p_{1}$ together with the transition $i, i^{\prime} \rightarrow j$ is enough to this end. The particular $\Gamma_{i, i^{\prime} \rightarrow j}^{d d d}$ contribution can be further simplified by the identification of a part of the integral in Eqs. (3.16) and (3.17), with the Lindhard function (details can be found in [42]).

Finally, the expressions for $\Gamma_{i, i^{\prime} \rightarrow j}^{\alpha \beta \delta}$ with $\alpha \beta \delta \neq d d d$, can be found in Appendix A. For the first order contribution (in the nuclear residual interaction, $V^{N N}$ ) to the RPA, which from now on is named as $R P A 1$, we can write,

$$
\Gamma_{i, i^{\prime} \rightarrow j}^{R P A} 1=\sum_{\alpha \beta \delta}\left(\Gamma_{i, i^{\prime} \rightarrow j}^{\alpha \beta \delta, l}+\Gamma_{i, i^{\prime} \rightarrow j}^{\alpha \beta \delta, u}\right) .
$$

The final step is to show the expressions for the spectra. In the present contribution the twobody induced decay is not evaluated. Then, it is convenient to re-write Eqs. (2.6)-(2.10), within the first order contribution to the RPA as the only FSI,

$$
\begin{aligned}
& N_{n}=2 \bar{\Gamma}_{n}+\bar{\Gamma}_{p}+\sum_{i, i^{\prime} ; j} N_{j(n)} \bar{\Gamma}_{i, i^{\prime} \rightarrow j}^{R P A 1}, \\
& N_{p}=\bar{\Gamma}_{p}+\sum_{i, i^{\prime} ; j} N_{j(p)} \bar{\Gamma}_{i, i^{\prime} \rightarrow j}^{R P A 1}, \\
& N_{n n}=\bar{\Gamma}_{n}+\sum_{i, i^{\prime} ; j} N_{j(n n)} \bar{\Gamma}_{i, i^{\prime} \rightarrow j}^{R P A}, \\
& N_{n p}=\bar{\Gamma}_{p}+\sum_{i, i^{\prime} ; j} N_{j(n p)} \bar{\Gamma}_{i, i^{\prime} \rightarrow j}^{R P A 1}, \\
& N_{p p}=0,
\end{aligned}
$$

where the normalization is now, $\bar{\Gamma} \equiv \Gamma / \Gamma_{1}$. These are already explicit expressions for the $N_{N}$ and $N_{N N}$-spectra, once some step functions limiting the range of the momentum (or angle) of the final particles are incorporated in Eqs. (3.3), (3.16) and (3.17). Also an additional step function is required if an energy threshold is considered. It should be noted that the RPA 1, together with any other contribution in which the nuclear residual interaction appears in first order (or in any odd order), is an interference term. The diagrams where the nuclear residual interaction is present an even number of times, has both interference terms and decay widths, being the decay widths the ones where the cut is in the middle of the diagram. In the next section we present numerical results for these expressions.

\section{Results and discussion}

In this section we present the numerical results for the $N_{n}, N_{p}, N_{n n}$ and $N_{n p}$-spectra using the first order contribution to the RPA. The LDA allows us to discuss the ${ }_{\Lambda}^{12} \mathrm{C}$ hypernucleus. The transition potential is represented by the exchanges of the $\pi, \eta, K, \rho, \omega$ and $K^{*}$-mesons, which formulation has been taken from [19] and the values of the different coupling constants and cutoff parameters appearing in the transition potential have been taken from [45]. For the nuclear 

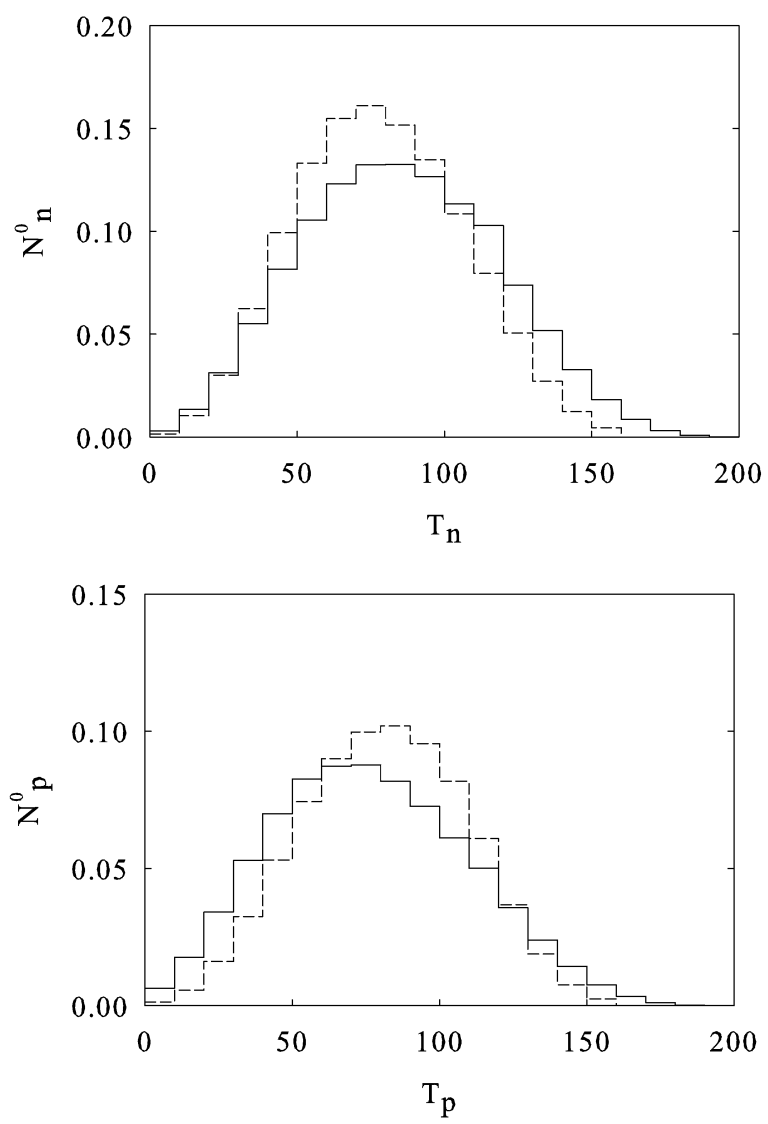

Fig. 6. $N_{n}$ and $N_{p}$-free kinetic energy spectra for ${ }_{\Lambda}^{12} \mathrm{C}$. The continuous line is our result, while the dashed line represents the INC one. The energy is given in MeV and the spectra have been evaluated using $10 \mathrm{MeV}$ steps for the kinetic energy. We have used $\Gamma_{n}=0.267$ and $\Gamma_{p}=0.936$, given in units of $\Gamma^{0}=2.52 \times 10^{-6} \mathrm{eV}$, where $\Gamma^{0}$ is the decay width of the $\Lambda$ in free space. For the evaluation of $\Gamma_{n, p}$, we have used Eq. (3.3) (together with Eqs. (3.1) and (3.2)), with the transition potential and parameters described in the first paragraph of the present section.

residual interaction, we have used the Bonn potential [46] in the framework of the parametrization presented in [47], which contains the exchange of $\pi, \rho, \sigma$ and $\omega$ mesons, while the $\eta$ and $\delta$-mesons are neglected. In implementing the LDA, the hyperon is assumed to be in the $1 s_{1 / 2}$ orbit of a harmonic oscillator well with frequency $\hbar \omega=10.8 \mathrm{MeV}$. As already stated, we have employed different values for the proton and neutron Fermi momenta, $k_{F_{n}}$ and $k_{F_{p}}$, respectively.

To start with, let us discuss the free spectra, that is, the spectra without final state interaction. To this end in Eqs. (3.21)-(3.25) all RPA 1-terms are eliminated. For the free spectra, the INC and the microscopic scheme should give the same result. Therefore, we make a comparison of our values with those of the INC, in order to test our values for the spectra. From a technical point of view, both methods evaluate the spectra differently. While in the INC, the emitted particles are classified according to their energies (or relative angles), in the present microscopic model the integral which represents the decay width is partitioned in energy (or relative angles) regions. These two procedures are equivalent. However, some differences show up, which are explained soon in this section. In Fig. 6 we present our result for the $N_{n}$ - and $N_{p}$-spectra and compare it 


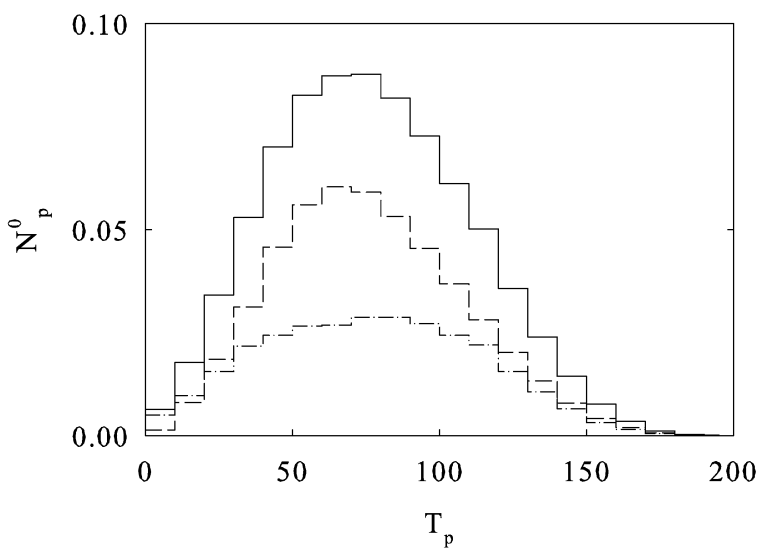

Fig. 7. Isospin components to the $N_{p}$-free kinetic energy spectra. The dashed line is the contribution where a proton is attached to the $\Lambda$-decay vertex, the dotted-dashed line represents the contribution where a neutron is attached to the same vertex, while the continuous line is the total contribution. Units are the same as in Fig. 6.

with the corresponding INC-result from [41]. Related to the INC, we notice that our value for the $N_{n}$-maximum is shifted towards a higher energy, while the opposite occurs for $N_{p}$. In fact, this shift is more marked in $N_{p}$.

To understand the origin of this behavior in Fig. 7 we have split $N_{p}$ into their two isospin components. The first isospin component is the charge-exchange one, where there is a proton in the $\Lambda$-vertex (that is, in the $p_{1}$-position in Fig. 3 ). The second is the charge-conserving contribution, which has a neutron in the just mentioned place. The first contribution is multiplied by a factor four (due to the isospin), while we have a factor one for the charge-conserving term. This makes the first contribution to be the dominant one. Notice that the transition potential is not the same for both contributions, because only the isovector terms in the transition potential act for the charge-exchange contribution. In addition, our results show that the particle attach to the $\Lambda$-vertex is slower than the other particle. This fact has a simple physical interpretation. In the expression for $\Gamma_{p}$ (or $\Gamma_{n}$ ), we take the principal value for the mesons propagators in the transition potential (note that the pole contribution in the pion propagator gives the mesonic decay width). Therefore, the pion pole is excluded, but as we are getting closer to it, the energy carried by the transition potential is of the order of the pion mass. In this case, the energy left to the $p_{1}$-particle becomes small, while the $p_{i}$-energy is the one carried by the transition potential. As the weight of the pion propagator is more important in the proximity of the pole, this favors the just described energy distribution. Going back to the comparison with the INC, the discrepancy is a consequence that for the available results this model makes no distinction between the inner isospin components (a fact that is not mandatory in the INC). We have implemented an isospin average to get rid of this difference, but we do not reproduce these results as they simply show the agreement between both methods.

We turn now to the inclusion of FSI. In the present contribution, we have limited the FSI to the first order contribution to the RPA. In Table 1, we present our results for $\Gamma_{i, i^{\prime} \rightarrow j}$, within three different approximations: the first order contribution (in the nuclear residual interaction) to the RPA (RPA 1), the same first order term to the ring series (ring 1), and the full ring approximation taken from [42]. The values quoted in this table result from the integration of the spectra over the whole energy region. The difference between $R P A 1$ and ring 1 is the inclusion of the exchange 
Table 1

Numerical results for $\Gamma_{i, i^{\prime} \rightarrow j}$, within several approximations. Note that $\Gamma_{n, p \rightarrow j}=\Gamma_{p, n \rightarrow j}$. The $R P A 1$ (ring 1)-line is the first order contribution to the RPA (ring), while the ring-line represents the total ring approximation take from [42]. All values are given in units of $\Gamma^{0}$

\begin{tabular}{lllll}
\hline Approximation & $\Gamma_{n, n \rightarrow n n}$ & $2 \Gamma_{n, p \rightarrow n n}$ & $2 \Gamma_{n, p \rightarrow n p}$ & $\Gamma_{p, p \rightarrow n p}$ \\
\hline$R P A 1$ & 0.0076 & 0.0096 & 0.0064 & 0.0994 \\
ring 1 & 0.0066 & 0.0090 & 0.0068 & 0.0895 \\
ring & 0.0261 & 0.0391 & 0.0371 & 0.1310 \\
\hline
\end{tabular}

terms. For $\Gamma_{n, n \rightarrow n n}$, the exchange terms increase this quantity by $\sim 14 \%$, while this percentage is $\sim 11 \%$ for $\Gamma_{p, p \rightarrow n p}$. Although they are different quantities, it is interesting to comment that the exchange terms produce also an increase of $\sim 34 \%$ and $\sim 30 \%$ in $\Gamma_{n}$ and $\Gamma_{p}$, respectively [24]. For the two remainders terms, we have an increase (decrease) of $\sim 5 \%(\sim 30 \%)$ for $\Gamma_{n, p \rightarrow n n}$ $\left(\Gamma_{n, p \rightarrow n p}\right)$.

The next point is to make an estimation of the accuracy of the first order RPA-contribution to account for the full RPA series. To this end, we compare the full ring series with ring 1 . We can see that only for $\Gamma_{p, p \rightarrow n p}$, the ring 1 is an adequate approximation, while there is a strong discrepancy for all the others terms. The reason for this behavior relays upon the inner isospin structure of the ring (or RPA) approximation. As already stated, the $\Gamma_{p, p \rightarrow n p}$ is dominated by graphs where a proton is attached to the $\Lambda$-weak decay vertex. The particle-hole bubbles (which propagates as the ring series), are built up from a neutron particle and a proton hole. The chargeconservation does not allow any other particle-hole configuration. The situation is different for $\Gamma_{n, n \rightarrow n n}, \Gamma_{n, p \rightarrow n n}, \Gamma_{n, p \rightarrow n p}$ and the $\Gamma_{p, p \rightarrow n p}$-contribution with a neutron in the $\Lambda$-vertex. As an example, we consider the second order contribution to $\Gamma_{n, n \rightarrow n n}$. This contribution has three particle-hole bubbles. The upper and lower bubbles can be only a neutron particle-neutron hole configuration. But the intermediate bubble can be either a neutron particle-neutron hole or a proton particle-proton hole one. Higher order contributions can have more complex configurations (for details, see [42]). In general terms, the convergence of the ring series is fast only when the particle-hole bubbles are of the same kind.

Also from Table 1, it is observed that the first order exchange contribution (which results from the difference between RPA 1 and ring 1), is smaller than the sum of all the remaining ring terms (which results from the difference between ring and ring 1). In spite of this, the preference for the $R P A 1$ over the ring approximation is based on the following point. The ring approximation can be used instead of the full RPA, as long as we have some knowledge on the exchange terms. The first order exchange terms are different for each isospin configuration. This does not allow us to draw any conclusion about the magnitude of the higher order exchange terms required to build up the RPA, because the isospin structure of higher order terms is different, as discussed in the above paragraph. From this analysis and our numerical values, it is clear that the ring approximation result is quite uncertain (due to our ignorance on the magnitude of the higher order exchange terms). From all these points, it is concluded that the RPA 1 is not an approximation to the full RPA, but it should be seen as a model in itself.

We analyze now the effect of the FSI on the spectra, where the free term has been subtracted. This is done in Figs. 8 and 9, for the RPA 1-contribution to the single and double kinetic energy spectra, respectively. Let us recall that within the RPA 1-model, all FSI are quantum interference terms. The $\Gamma_{n, n \rightarrow j}$ and $\Gamma_{p, p \rightarrow j}$ contributions have $i=i^{\prime}$ and for convenience are called as diagonal interference terms, while the $\Gamma_{n, p \rightarrow j^{-}}$and $\Gamma_{p, n \rightarrow j}$-ones have $i \neq i^{\prime}$ and are named as non-diagonal interference terms. It should be noted that the $\bar{\Gamma}_{i, i^{\prime} \rightarrow j}$-functions can be 

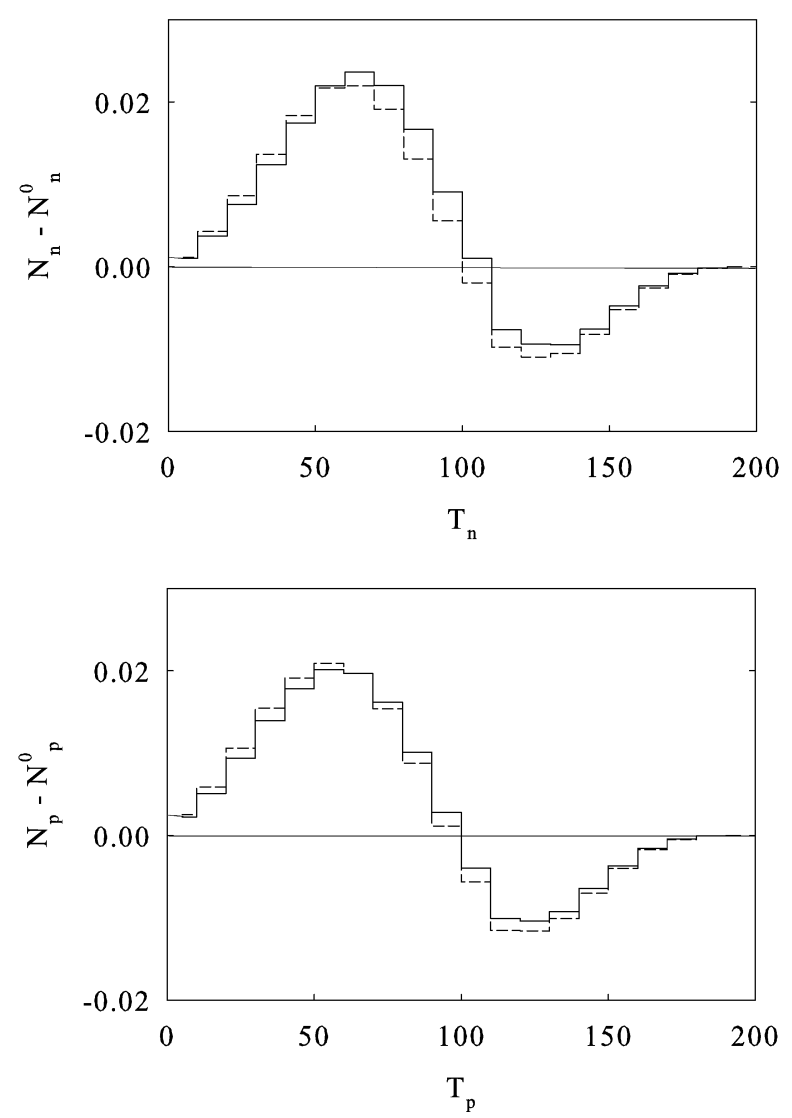

Fig. 8. First order RPA contribution to the single nucleon kinetic energy spectra for ${ }_{\Lambda}^{12} \mathrm{C}$. For convenience, the free spectra have been subtracted. The continuous line is our final RPA 1-result and the dashed line represents the result without non-diagonal interference terms.

either positive or negative. We present values with and without the non-diagonal interference terms. Throughout this section we pay much attention to study the relative importance of the non-diagonal interference terms. Certainly, the possibility of neglecting these terms would simplify the calculation. In Fig. 8, a typical RPA-behavior is shown. From Table 1, together with Eqs. (3.21)-(3.25), the non-diagonal interference terms increase the sum of $\left(N_{n}-N_{n}^{0}\right)$ and $\left(N_{p}-N_{p}^{0}\right)$, in $\sim 18 \%$ and $\sim 7 \%$, respectively.

In Fig. 9 a similar analysis is done for the double kinetic energy spectra. Here, the nondiagonal interference term increases $\left(N_{n p}-N_{n p}^{0}\right)$ in $\sim 6 \%$, while the effect over $\left(N_{n n}-N_{n n}^{0}\right)$ is very important: it increases the result in more than a factor of two. To understand these factors, we refer again to Table 1 . Among all the $\Gamma_{i, i^{\prime} \rightarrow j}, \Gamma_{p, p \rightarrow n p}$ is the dominant one. Non-diagonal interference terms are the next contribution in magnitude, but they are one order of magnitude smaller. Only for $N_{n n}$, the $\Gamma_{p, p \rightarrow n p}$ contribution is not present. In this case, the non-diagonal interference term becomes very important. Once more, the reason for this behavior relays upon the isospin factors. A charge-exchange isospin vertex has a factor $\sqrt{2}$, in comparison with a factor one for the charge-conserving. And within our approximation, charge-exchange contributions are only present in $\Gamma_{p, p \rightarrow n p}$. 

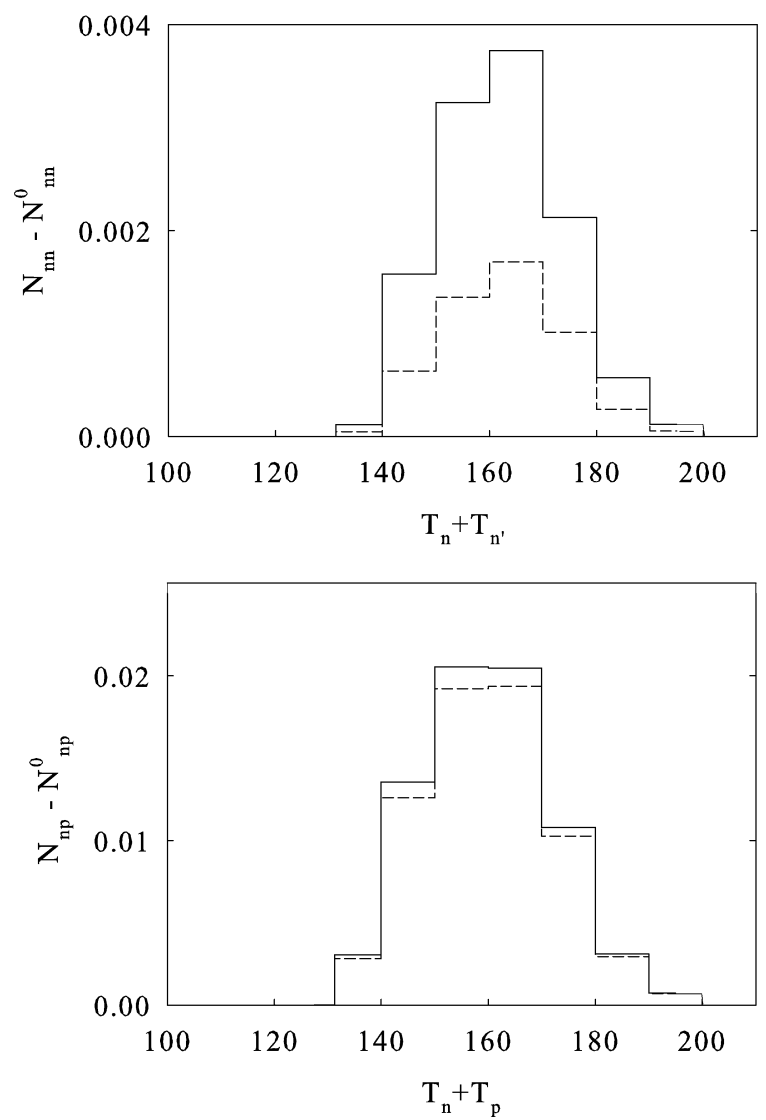

Fig. 9. The same as Fig. 8, but for the double-nucleon spectra.

In Fig. 10, we present our final result for the single kinetic energy spectra $N_{n}$ and $N_{p}$ and we make a comparison with data. In this figure, the free spectra are also shown. The RPA 1approximation improves the free result, as it reduces the spectra in the high energy region, while it produces an increase at low energies. However, it is clear that some important FSI are missing. The comparison of our results with data in the low energy region gives us an indication of these missing terms. The Goldstone diagram used to evaluate the free spectra (see Fig. 3), has a two particles-one hole $(2 p 1 h)$ configuration. In fact, we have one particle attached to the $\Lambda$-weak decay vertex, plus a one particle-one hole bubble $(1 p 1 h)$, attached to the strong vertex. The $R P A 1$ adds basically configurations restricted to $1 p 1 h$-bubbles (plus the corresponding exchange terms). The next RPA-order or even the full RPA, is always restricted to this phase space. It is likely that the required increase in phase space would be obtained by the addition of second order self-energy configurations. Our knowledge from electron scattering, is that the self-energy opens the $3 p 2 h$-decay channel and moves intensity towards the low energy region. The inclusion of this kind of contribution is beyond the scope of the present contribution. In the same figure, we present our RPA 1-results with SRC in the nuclear residual interaction (for details about SRC, see [42] and references therein). This result is discussed soon. 

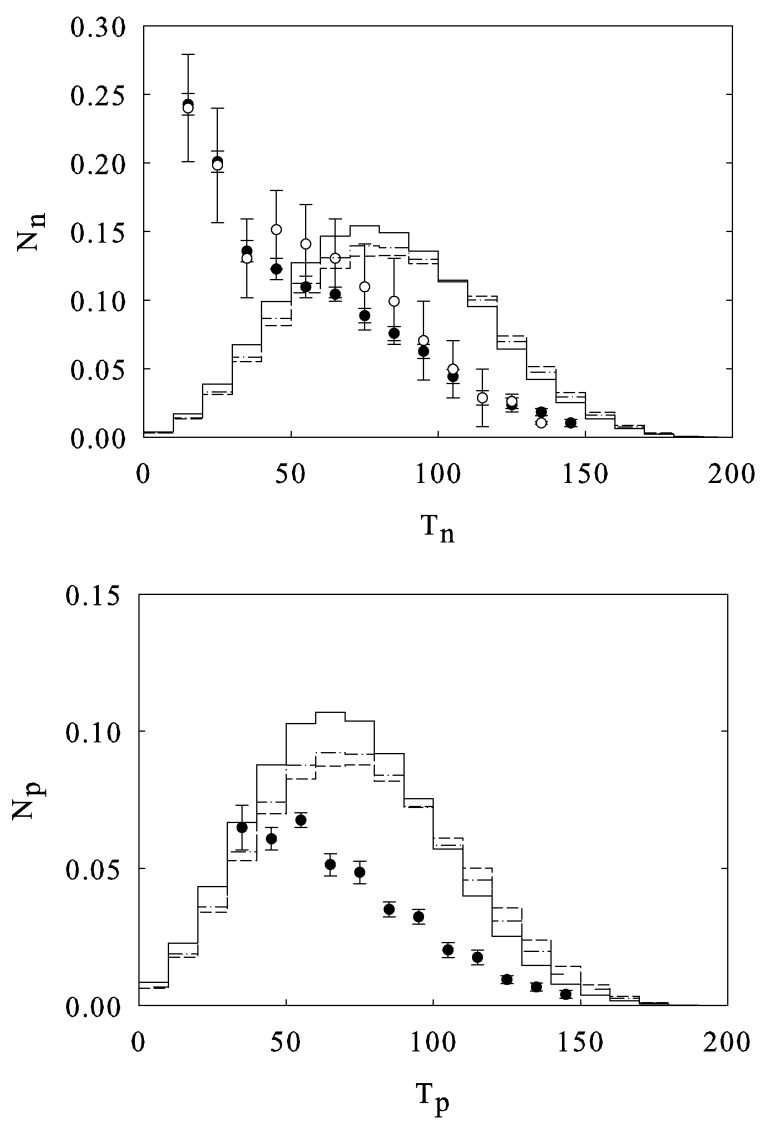

Fig. 10. Final result of the $N_{n}$ and $N_{p}$ kinetic energy spectra for ${ }_{\Lambda}^{12} \mathrm{C}$. The continuous (dot-dashed) line is our RPA 1-result without (with) SRC in the nuclear residual interaction and the dashed line represents the free result. Data are from KEK-E369 [6] (not-filled circles) and KEK-E508 [7] (filled circles).

In Fig. 11, the double kinetic energy spectra are shown together with the free spectra. In this case, the RPA 1-approximation does not affect the spectra very much. Finally, in Fig. 12, the opening angle distribution of $n n$ and $n p$ pairs is compared with data. Also in these figures we present both the free and the RPA 1-results. These figures give us further evidence of the importance of including $3 p 2 h$-final states. The two emitted particles from the $2 p 1 h$ final state, emerge from the nuclei mainly with back-to-back angles. The reason is the following: from momentum conservation, we have $\boldsymbol{k}+\boldsymbol{h}_{i}=\boldsymbol{p}_{1}+\boldsymbol{p}_{i}$ (see Fig. 3). The $\Lambda$-wave function is peaked at $|\boldsymbol{k}|=0$, while $\left|\boldsymbol{h}_{i}\right|$ ranges from 0 up to $k_{F}$. In the particular case when $|\boldsymbol{k}|=\left|\boldsymbol{h}_{i}\right|=0$, we have $\boldsymbol{p}_{1}=-\boldsymbol{p}_{i}$, which represents the extreme case of back-to-back kinematics. The kinematical conditions are very different for the $3 p 2 h$-states, which allows the existence of any angle between two of the three outgoing particles.

Going back to Fig. 12, the data shows intensity in forward angles, which from the theoretical point of view would required final states like the $3 p 2 h$-mentioned ones. It is clear also that not only the distribution, but also the area does not match. The origin of this discrepancy can be understood from Fig. 10. In accordance with the data, our spectra (in Fig. 12), have been 

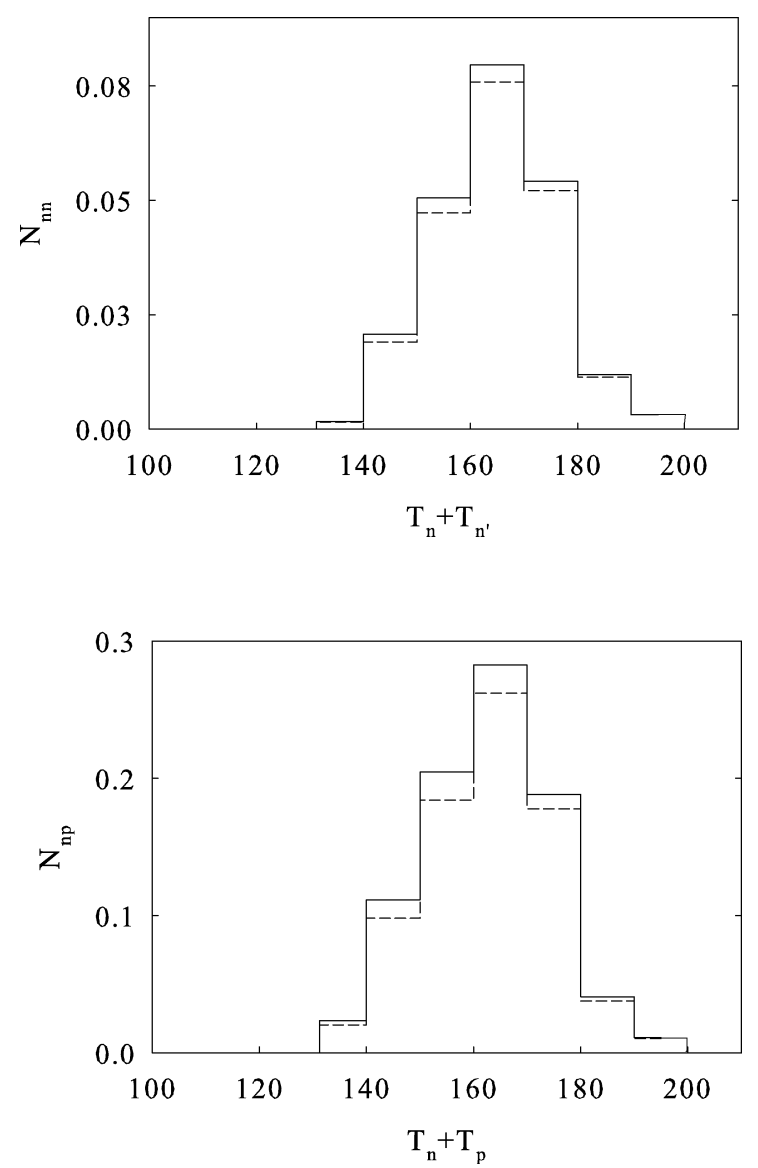

Fig. 11. Final result of the $N_{n n}$ and $N_{n p}$ kinetic energy spectra for ${ }_{\Lambda}^{12} \mathrm{C}$. The continuous line is our RPA 1-result without $\mathrm{SRC}$ in the nuclear residual interaction and the dashed line represents the free result.

evaluated using an energy threshold of $30 \mathrm{MeV}$ for both protons and neutrons and our complete spectra (in Fig. 10), have very little intensity for energies smaller than the threshold, while data suggests that the theoretical spectra should move towards lower energies. In spite of this, and due to the big error bars, we believe that this point requires much more efforts from both the theoretical and the experimental point of view.

In Table 2, we show our values for $N_{n} / N_{p}$ and $N_{n n} / N_{n p}$, which result from the integration of the corresponding spectra over the whole energy region. The objective of this table is to make a comparison of the RPA 1-result with the full ring series and with ring 1. For this reason, no energy threshold has been implemented, as this would affect the result, making the analysis of the different approximations more difficult. In the first line, we show the results without FSI, where the values quoted as 'dir', refer to the first diagram in Fig. 3, while the complete result is the sum of both diagrams (direct plus exchange), in the same figure. ${ }^{1}$ From this table, we see

\footnotetext{
1 To avoid confusion, it is worth to mention that the word 'exchange' has been used for two different contributions: the plain use of 'exchange' refers to the Pauli exchange term, while by 'charge-exchange' we indicate an isospin component.
} 

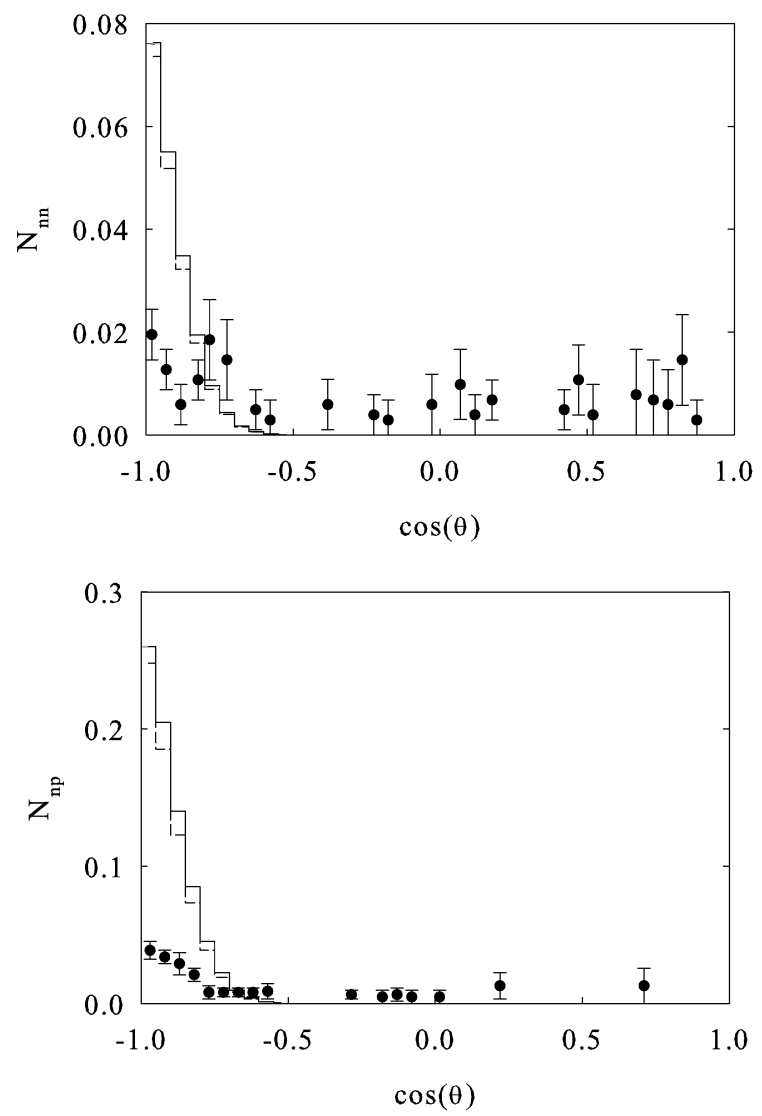

Fig. 12. Opening angle distribution of $n n$ and $n p$ pairs. The continuous line is our RPA 1-result without SRC in the nuclear residual interaction and the dashed line represents the free result. Data are from [11].

Table 2

The ratios $N_{n} / N_{p}$ and $N_{n n} / N_{n p}$ for ${ }_{\Lambda}^{12} \mathrm{C}$ hypernucleus. Within parenthesis we present our results without the nondiagonal interference terms. The index 'dir' indicates the values without exchange terms. The line named as ring, represents the full ring series, while the direct part in line RPA 1, is called ring 1 as in the text. The ring values have been taken from Tables 2 and 3 of [42]

\begin{tabular}{|c|c|c|c|c|}
\hline Ref. & $N_{n} / N_{p}$ & {$\left[N_{n} / N_{p}\right]^{\operatorname{dir}}$} & $N_{n n} / N_{n p}$ & {$\left[N_{n n} / N_{n p}\right]^{\operatorname{dir}}$} \\
\hline LDA, no-FSI & 1.571 & 1.458 & 0.285 & 0.229 \\
\hline LDA with $R P A 1$ & $1.545(1.530)$ & & $0.273(0.265)$ & \\
\hline LDA with ring 1 & & $1.445(1.430)$ & & $0.222(0.215)$ \\
\hline LDA with ring [42] & & $1.514(1.459)$ & & $0.257(0.229)$ \\
\hline
\end{tabular}

that exchange terms are important, specially for the free case. The RPA 1 has a small influence. In spite of this fact, it should be mentioned that the non-diagonal interference terms represents around half of the FSI-contribution. We observe that the RPA 1 decreases instead of increasing, the free value. The opposite situation occurs with the full ring series. This behavior is understood as a consequence of the absence (presence) of charge-exchange terms in $\Gamma_{i, i^{\prime} \rightarrow j}$ with $i, i^{\prime} \neq$ $p, p$, in RPA 1 (ring approximation). Moreover, the ring approximation leaves these ratios almost 
Table 3

The ratios $N_{n} / N_{p}$ and $N_{n n} / N_{n p}$ for ${ }_{\Lambda}^{12} \mathrm{C}$ hypernucleus. The index 'diag', indicates the values without the quantum interference terms between the $(\Lambda n \rightarrow n n)$ and $(\Lambda p \rightarrow n p)$-amplitudes. In accordance with data, for $N_{n} / N_{p}$ we have used an energy threshold of $60 \mathrm{MeV}$ for both protons and neutrons, while the reported values for $N_{n n} / N_{n p}$, correspond to angles which satisfy $\cos (\theta)<-0.80$, together with an energy threshold of $30 \mathrm{MeV}$ (for both protons and neutrons)

\begin{tabular}{|c|c|c|c|c|}
\hline Ref. & $N_{n} / N_{p}$ & $\left(N_{n} / N_{p}\right)^{\operatorname{diag}}$ & $N_{n n} / N_{n p}$ & $\left(N_{n n} / N_{n p}\right)^{\operatorname{diag}}$ \\
\hline LDA, no-FSI & 1.791 & & 0.279 & \\
\hline LDA with $R P A 1$ & 1.863 & 1.858 & 0.272 & 0.267 \\
\hline KEK-E508 [7] & $2.00 \pm 0.09 \pm 0.14$ & & & \\
\hline KEK-E508 [8] & & & $0.40 \pm 0.09 \pm 0.04$ & \\
\hline
\end{tabular}

unchanged (with respect with the free result) when the non-diagonal interference terms are not considered, while their inclusion have more effect than in RPA 1 (or ring 1). As a further comment from this table, it is clear that the magnitude of the exchange terms is important enough to object the use of the ring series.

In Table 3, we show our final results for $N_{n} / N_{p}$ and $N_{n n} / N_{n p}$, and compare them with data. For $N_{n} / N_{p}$, we have used an energy threshold of $60 \mathrm{MeV}$ for both protons and neutrons, in accordance with [7]; while the results for $N_{n n} / N_{n p}$ correspond to angles between the outgoing particles, for which $\cos (\theta)<-0.80$ and an energy threshold of $30 \mathrm{MeV}$ [8]. The increase in $N_{n} / N_{p}$, when compared with the results in Table 2 , is easily understood in terms of the discussion done in Fig. 6: The dominant charge-exchange term in $N_{p}^{0}$ makes the maximum in the protoninduced spectra to be placed at a smaller energy position than the neutron-induced one. It should be mentioned that we present this table for completeness. Our good result for $N_{n} / N_{p}$ could be misinterpreted. The INC is used for two purposes: the theoretical prediction of the spectra and the extraction of the so-called experimental value for the $\Gamma_{n} / \Gamma_{p}$-ratio. For the second point, certain coefficients are evaluated which, together with data for $N_{n} / N_{p}$ (or $N_{n n} / N_{n p}$ ), are used to obtained $\left(\Gamma_{n} / \Gamma_{p}\right)^{\exp }$ (for details, see [39] and [40]). Within the INC, the comparison between the theoretical prediction for $N_{n} / N_{p}$ and $N_{n n} / N_{n p}$, with data makes much sense. Our point of view is different: our final aim is the reproduction of the experimental nucleon spectra. The value for $\Gamma_{n} / \Gamma_{p}$ would be the one predicted by our model, as the reproduction of the spectra would produce an exact match between the theoretical and the experimental ratio, by definition. Clearly, the determination of the ratio $\Gamma_{n} / \Gamma_{p}$ is important because it tests our knowledge of the baryonbaryon strangeness-changing weak interactions, which is one of the main motivations for these studies. We believe that it is not possible to disentangle completely this issue from the many-body problem which is intricate by itself.

As a final point for this section and beyond the limitations in phase space in the RPA, the nuclear residual interaction employed in the RPA, should be discussed. To perform this calculation we have chosen the Bonn potential where we have neglected SRC in all results except for the ones in Fig. 10. From this figure, we can see that the addition of SRC reduce significantly the $R P A$ 1-contribution. The interaction without SRC, has been selected to explore the RPA results under extreme conditions. The final outcome shows us that the RPA does not alter the free spectra very much. The addition of SRC makes this contribution even less important. From this, we can conclude that the RPA 1 is adequate enough to explore the RPA contribution and from our whole analysis, it is clear that the RPA does not represent the relevant kind of FSI required to describe the $\Lambda$-weak decay spectra. From the INC we know that the FSI are important, which states our problem as the finding of the most significant diagrams to account for the FSI. We do not known a priori the answer to this question. To obtain some insight about this problem, 
the RPA has been a good starting point due to its simplicity. The search of the relevant set of diagrams has an additional difficulty, which is the selection of the nuclear residual interaction, together with the adjustment of some potential-parameters. The Bonn potential is one possible choice, but the truth is that we do not know the strong interaction in this energy-momentum region. However, the INC provides us with a powerful tool to settle the strong interaction: in our microscopic scheme it is easy to turn off the interference terms not contained in the INC. Under these conditions, and once the adequate set of diagrams are found, the INC-result can be used to fix the strong interaction. Note that the INC does not employ an effective strong interaction but the experimental scattering cross section between nucleons. This would give us some confidence on the strong interaction so adjusted. For this particular purpose, the use of the INC is better than the data themselves, because the theoretical model for the weak transition potential would be the same in both the INC and in our microscopic model. Afterwards, the interference terms should be added and a comparison with data should be done. In our opinion, it is this whole schedule the one which should be employed to test our knowledge of the baryon-baryon strangenesschanging weak interactions. The present contribution is a step forward in this direction. In [42], we have presented our scheme in general terms. That model has been developed in the present work, but for a particular kind of FSI. In a forthcoming work, the $3 p 2 h$-decay channel which is not contained in the RPA and can be represented by second order self-energy contributions, will be included. At variance with the present calculation, this kind of FSI contains both decay widths and interference terms.

\section{Conclusions}

The present work addresses the problem of the theoretical interpretation of the spectra of nucleons emitted from the non-mesonic weak decay of a $\Lambda$-hypernucleus. To the best of our knowledge, we have presented for the first time a scheme which deals with this issue using a microscopic formalism. We have presented results for the single and double kinetic energy spectra and also for the opening angle distribution of $n n$ and $n p$ pairs. The FSI have been incorporated by means of the first order contribution (in the nuclear residual interaction) to the RPA. Our formalism naturally contains the quantum interference terms between any pair of decay amplitudes which end in the same final state. The quantum interference terms appear once the FSI are incorporated. From the INC we know that the FSI are important. In a microscopic scheme there is a huge amount of possible diagrams which constitute these FSI. Beforehand, we do not known which set of diagrams is the most significant one. The RPA has been considered as a starting point in this analysis because it simplicity gives us some insight on the kind of the contributions required. However, the RPA itself does not represent an important contribution. The comparison of our results with data suggests that the FSI should connect $2 p 1 h$ with $3 p 2 h$ configurations. This kind of study is much more complex and it is beyond the scope of the present contribution.

We have payed a particular attention to the non-diagonal interference terms which are important within the RPA. In fact, they are very significant in the $N_{n n}$-spectra, while they can be neglected in $N_{n p}$. The effect on the $N_{n}$-spectra is a moderate increase and it is also small for the $N_{p}$-spectra. The origin of this behavior does not relay upon any particularity of the RPA, but on some isospin factors. We agree that only when the relevant FSI-diagrams are found and incorporated, we would be able to know if the quantum interference terms are important or not. But from our results, it is clear that they cannot be ignored.

The existing and more successful formalism which takes care of the same problem is the INC. The INC is a semi-phenomenological approach, where the trajectory of the particles emitted 
in the weak decay is tracked in their way out of the nucleus. The FSI are incorporated in the INC using free path and experimental cross sections between nucleons. Unfortunately, it is not possible to establish a biunique relation between the processes in the INC and in our microscopic model. The limitation of our microscopic model is the technical difficulty in finding the relevant FSI-diagrams. However, our scheme improves the INC in two main points. The first one is the incorporation of the quantum interference terms mentioned above. The second one refers to the description of light hypernuclei, where the employment of the INC is not appropriate. We have presented our results using nuclear matter together with the LDA, which is also a bad approximation for light hypernuclei. However, our expressions are not limited to nuclear matter and they can be used in a finite nucleus calculation. The incorporation of FSI in this kind of work is certainly a quite involve task. Our aim is to describe the nuclear spectra of medium hypernuclei where the use of nuclear matter is possible. However, once this goal is eventually accomplished, the knowledge of the relevant FSI-diagrams would encourage the evaluation of a finite nucleus calculation for light hypernuclei.

We would like to comment now on the $\Gamma_{n} / \Gamma_{p}$-ratio. An experimental determination of this ratio suffers from two ambiguities: the possible contribution of two-body induced non-mesonic weak decay (which are originated from ground state correlations, GSC) and the effect of FSI. It is worth to mention here that these two processes should not be confused. The two-body induced, is part of the primary decay as shown in Eqs. (2.1)-(2.5). After the primary decay takes place, the strong interaction between nucleons (i.e., the FSI) starts acting. The FSI affects nucleons from both one- and two-body induced decays. A final $3 p 2 h$-state, for example, can be originated either from a GSC (without FSI) or from the action of FSI over a one-body induced decay. Moreover, there is an interference term between these processes, as the initial and final state is the same. This interference term cannot be evaluated within the INC as it is a pure quantum mechanic effects. Beyond technical difficulties, this kind of contributions are formally part of the $\Gamma_{i, i^{\prime} \rightarrow j}$ in Eqs. (2.6)-(2.10). This is just one example, the theoretical problem of the inclusion of both GSC and FSI, is certainly a very difficult problem. Any experimental determination of the $\Gamma_{n} / \Gamma_{p}$-ratio, assumes a model for this theoretical problem.

Referring to the INC, it is used to predict nucleon spectra and to extract the so-called experimental value for the $\Gamma_{n} / \Gamma_{p}$-ratio. For the extraction of the ratio, the experimental value for $N_{n} / N_{p}$ or $N_{n n} / N_{n p}$ (together with the corresponding error bars) is used as an input. To put it in simple terms, a typical theoretical value for the ratio is, $\Gamma_{n} / \Gamma_{p} \sim 0.3$, while data analyzed by means of the INC, gives a result $\left(\Gamma_{n} / \Gamma_{p}\right)$ exp $\sim 0.4 \pm 0.1$ (we have focused on the $\cos (\theta)<-0.80$ region, for ${ }_{\Lambda}^{12} \mathrm{C}$ ). Based on this analysis, it is reasonable to assert that the $\Gamma_{n} / \Gamma_{p}$-puzzle has been solved. However, one should be aware of the fact that the experimental information used as an input in the INC is the $N_{n} / N_{p^{-}}$(or $N_{n n} / N_{n p^{-}}$)ratio, where here $N_{N}$ (or $N_{N N}$ ) is the integral of the spectra over energy or angle. Clearly, this integration erase some physical information. In fact, while the INC reproduce fairly well the $N_{n}$-kinetic energy spectra, some discrepancies remains for the $N_{p}$-kinetic energy spectra. Having in mind this element, together with the above mentioned ambiguities, it is our opinion that the real theoretical problem is the reproduction of the spectra. From the present contribution, we have indications that the quantum interference terms should be included in the spectra evaluation. The next step, is to found the relevant FSI-diagrams. As discussed, the RPA 1-results has given us some hints on this issue. 


\section{Acknowledgements}

I would like to thank F. Krmpotić, G. Garbarino and A. Ramos, for fruitful discussions and for the critical reading of the manuscript. This work has been partially supported by the CONICET, under contract PIP 6159.

\section{Appendix A}

In this appendix we present the explicit form for $\Gamma_{i, i^{\prime} \rightarrow j}^{\alpha \beta \delta}$ with $\alpha \beta \delta \neq d d d$. In order to simplify the expressions, all functions which have the isospin $\tau-\left(\tau^{\prime}-\right)$ index, carries an energymomentum $q\left(q^{\prime}\right)$, while the nuclear strong interaction has an isospin index $\tau_{N}$ and has a $t$-energy-momentum transfer. Moreover, as each function is shown in a separate subsection, the super-index $\alpha \beta \delta$ is written only when it is necessary.

\section{A.1. The $\Gamma_{i, i^{\prime} \rightarrow j}^{\text {ded }}$-contribution}

The $\mathcal{S}_{\tau^{\prime} \tau_{N} \tau}^{\text {ded }}\left(q, q^{\prime}, t\right)$ function is

$$
\begin{aligned}
\mathcal{S}_{\tau^{\prime} \tau_{N} \tau}^{\text {ded }}\left(q, q^{\prime}, t\right)= & 4 \mathcal{V}_{C, \tau_{N}}\left\{S_{\tau^{\prime}} S_{\tau}+S_{\tau^{\prime}}^{\prime} S_{\tau}^{\prime}+2 S_{V, \tau^{\prime}} S_{V, \tau}+P_{C, \tau^{\prime}} P_{C, \tau}\right. \\
& \left.+3 P_{\sigma, \tau^{\prime}} P_{\sigma, \tau}+P_{L, \tau^{\prime}} P_{L, \tau}+P_{L, \tau^{\prime}} P_{\sigma, \tau}+P_{\sigma, \tau^{\prime}} P_{L, \tau}\right\} \\
& +4 \mathcal{V}_{\sigma, \tau_{N}}\left\{3 S_{\tau^{\prime}} S_{\tau}-S_{\tau^{\prime}}^{\prime} S_{\tau}^{\prime}-2 S_{V, \tau^{\prime}} S_{V, \tau}+3 P_{C, \tau^{\prime}} P_{C, \tau}\right. \\
& \left.-3 P_{\sigma, \tau^{\prime}} P_{\sigma, \tau}-P_{L, \tau^{\prime}} P_{L, \tau}-2\left(P_{L, \tau^{\prime}} P_{\sigma, \tau}+P_{\sigma, \tau^{\prime}} P_{L, \tau}\right)\right\} \\
& +4 \mathcal{V}_{L, \tau_{N}}\left\{S_{\tau^{\prime}} S_{\tau}-\left(1+2(\hat{\boldsymbol{q}} \cdot \hat{\boldsymbol{t}})^{2}\right) S_{\tau^{\prime}}^{\prime} S_{\tau}^{\prime}-2(\hat{\boldsymbol{q}} \cdot \hat{\boldsymbol{t}})^{2} S_{V, \tau^{\prime}} S_{V, \tau}\right. \\
& +\left(-1+2(\hat{\boldsymbol{q}} \cdot \hat{\boldsymbol{t}})^{2}\right) P_{L, \tau^{\prime}} P_{L, \tau}+P_{C, \tau^{\prime}} P_{C, \tau}-P_{\sigma, \tau^{\prime}} P_{\sigma, \tau} \\
& \left.+\left(-1+2(\hat{\boldsymbol{q}} \cdot \hat{\boldsymbol{t}})^{2}\right) P_{\sigma, \tau^{\prime}} P_{L, \tau}\right\},
\end{aligned}
$$

where $q=k-p_{1}=q^{\prime}$ and $t=h_{i}-h_{i^{\prime}}$.

$$
\begin{aligned}
\Gamma_{n, n \rightarrow n n}^{l}= & \tilde{\Gamma}_{n, n \rightarrow n n, 111}^{l, n}+\tilde{\Gamma}_{n, n \rightarrow n n, 000}^{l, n}+\tilde{\Gamma}_{n, n \rightarrow n n, 110}^{l, n}+\tilde{\Gamma}_{n, n \rightarrow n n, 101}^{l, n}+\tilde{\Gamma}_{n, n \rightarrow n n, 011}^{l, n} \\
& +\tilde{\Gamma}_{n, n \rightarrow n n, 100}^{l, n}+\tilde{\Gamma}_{n, n \rightarrow n n, 010}^{l, n}+\tilde{\Gamma}_{n, n \rightarrow n n, 001}^{l, n}, \\
\Gamma_{n, n \rightarrow n n}^{u}= & \Gamma_{n, n \rightarrow n n}^{l}, \\
\Gamma_{n, p \rightarrow n n}^{l}= & 2\left(-\tilde{\Gamma}_{n, p \rightarrow n n, 111}^{l, n}-\tilde{\Gamma}_{n, p \rightarrow n n, 110}^{l, n}+\tilde{\Gamma}_{n, p \rightarrow n n, 011}^{l, n}+\tilde{\Gamma}_{n, p \rightarrow n n, 010}^{l, n}\right), \\
\Gamma_{n, p \rightarrow n n}^{u}= & 0, \\
\Gamma_{p, n \rightarrow n n}^{l}= & 0, \\
\Gamma_{p, n \rightarrow n n}^{u}= & 2\left(-\tilde{\Gamma}_{n, p \rightarrow n n, 111}^{u, n}+\tilde{\Gamma}_{n, p \rightarrow n n, 110}^{u, n}-\tilde{\Gamma}_{n, p \rightarrow n n, 011}^{u, n}+\tilde{\Gamma}_{n, p \rightarrow n n, 010}^{u, n}\right), \\
\Gamma_{n, p \rightarrow n p}^{l}= & 0, \\
\Gamma_{n, p \rightarrow n p}^{u}= & 2\left(-\tilde{\Gamma}_{n, p \rightarrow n p, 111}^{u, n}-\tilde{\Gamma}_{n, p \rightarrow n p, 110}^{u, n}+\tilde{\Gamma}_{n, p \rightarrow n p, 011}^{u, n}+\tilde{\Gamma}_{n, p \rightarrow n p, 010}^{u, n}\right), \\
\Gamma_{p, n \rightarrow n p}^{l}= & 2\left(-\tilde{\Gamma}_{n, p \rightarrow n p, 111}^{l, n}+\tilde{\Gamma}_{n, p \rightarrow n p, 110}^{l, n}-\tilde{\Gamma}_{n, p \rightarrow n p, 011}^{l, n}+\tilde{\Gamma}_{n, p \rightarrow n p, 010}^{l, n}\right), \\
\Gamma_{p, n \rightarrow n p}^{u}= & 0, \\
\Gamma_{p, p \rightarrow n p}^{l}= & \tilde{\Gamma}_{p, p \rightarrow n p, 111}^{l, n}+\tilde{\Gamma}_{p, p \rightarrow n p, 000}^{l, n}-\tilde{\Gamma}_{p, p \rightarrow n p, 110}^{l, n}+\tilde{\Gamma}_{p, p \rightarrow n p, 101}^{l, n}
\end{aligned}
$$




$$
\begin{aligned}
& -\tilde{\Gamma}_{p, p \rightarrow n p, 011}^{l, n}-\tilde{\Gamma}_{p, p \rightarrow n p, 100}^{l, n}+\tilde{\Gamma}_{p, p \rightarrow n p, 010}^{l, n}-\tilde{\Gamma}_{p, p \rightarrow n p, 001}^{l, n} \\
& +4\left(-\tilde{\Gamma}_{p, p \rightarrow n p, 111}^{l, p}+\tilde{\Gamma}_{p, p \rightarrow n p, 101}^{l, p}\right), \\
\Gamma_{p, p \rightarrow n p}^{l}= & \Gamma_{p, p \rightarrow n p}^{u} .
\end{aligned}
$$

\section{A.2. The $\Gamma_{i, i^{\prime} \rightarrow j}^{d d e}$ - and $\Gamma_{i, i^{\prime} \rightarrow j}^{e d d}$-contributions}

The $\mathcal{S}_{\tau^{\prime} \tau_{N} \tau}^{d d e}\left(q, q^{\prime}, t\right)$ function is

$$
\begin{aligned}
\mathcal{S}_{\tau^{\prime} \tau_{N} \tau}^{d d e}\left(q, q^{\prime}, t\right)= & 4 \mathcal{V}_{C, \tau_{N}}\left\{\left(\hat{\boldsymbol{q}} \cdot \hat{\boldsymbol{q}}^{\prime}\right)\left(S_{\tau^{\prime}} S_{\tau}+S_{\tau^{\prime}} S_{\tau}^{\prime}-2 S_{\tau^{\prime}} S_{V, \tau}\right)+P_{C, \tau^{\prime}} P_{C, \tau}\right. \\
& \left.+3 P_{C, \tau^{\prime}} P_{\sigma, \tau}+P_{C, \tau^{\prime}} P_{L, \tau}\right\} \\
& +4 \mathcal{V}_{\sigma, \tau_{N}}\left\{\left(\hat{\boldsymbol{q}} \cdot \hat{\boldsymbol{q}}^{\prime}\right)\left(S_{\tau^{\prime}}^{\prime} S_{\tau}^{\prime}+S_{\tau^{\prime}} S_{\tau}^{\prime}+2\left(S_{\tau^{\prime}}^{\prime} S_{V, \tau}+S_{\tau} S_{V, \tau^{\prime}}-S_{\tau}^{\prime} S_{V, \tau^{\prime}}\right)\right)\right. \\
& -3 P_{\sigma, \tau^{\prime}} P_{\sigma, \tau}+\left(-1+2\left(\hat{\boldsymbol{q}} \cdot \hat{\boldsymbol{q}}^{\prime}\right)^{2}\right) P_{L, \tau^{\prime}} P_{L, \tau}+3 P_{C, \tau} P_{\sigma, \tau^{\prime}} \\
& \left.+P_{C, \tau} P_{L, \tau^{\prime}}-\left(P_{\sigma, \tau^{\prime}} P_{L, \tau}+P_{L, \tau^{\prime}} P_{\sigma, \tau}\right)\right\} \\
& +4 \mathcal{V}_{L, \tau N}\left\{\left(\hat{\boldsymbol{q}} \cdot \hat{\boldsymbol{q}}^{\prime}\right)\left(S_{\tau^{\prime}}^{\prime} S_{\tau}^{\prime}+S_{\tau} S_{\tau^{\prime}}^{\prime}+2 S_{\tau^{\prime}}^{\prime} S_{V, \tau}\right)-P_{\sigma, \tau^{\prime}} P_{\sigma, \tau}\right. \\
& +\left(-1+2\left(\hat{\boldsymbol{q}} \cdot \hat{\boldsymbol{q}}^{\prime}\right)^{2}\right) P_{L, \tau^{\prime}} P_{L, \tau}+P_{C, \tau} P_{\sigma, \tau^{\prime}} \\
& \left.+P_{C, \tau} P_{L, \tau^{\prime}}+\left(-1+2\left(\hat{\boldsymbol{q}} \cdot \hat{\boldsymbol{q}}^{\prime}\right)^{2}\right) P_{\sigma, \tau^{\prime}} P_{L, \tau}-P_{L, \tau^{\prime}} P_{\sigma, \tau}\right\},
\end{aligned}
$$

where $q=k-p_{1}, t=k-p_{1}$ and $q^{\prime}=p_{1}-h_{i^{\prime}}$.

$$
\begin{aligned}
\Gamma_{n, n \rightarrow n n}^{l}= & \tilde{\Gamma}_{n, n \rightarrow n n, 111}^{l, n}+\tilde{\Gamma}_{n, n \rightarrow n n, 000}^{l, n}+\tilde{\Gamma}_{n, n \rightarrow n n, 110}^{l, n}+\tilde{\Gamma}_{n, n \rightarrow n n, 101}^{l, n}+\tilde{\Gamma}_{n, n \rightarrow n n, 011}^{l, n} \\
& +\tilde{\Gamma}_{n, n \rightarrow n n, 100}^{l, n}+\tilde{\Gamma}_{n, n \rightarrow n n, 010}^{l, n}+\tilde{\Gamma}_{n, n \rightarrow n n, 001}^{l, n}, \\
\Gamma_{n, n \rightarrow n n}^{u}= & \Gamma_{n, n \rightarrow n n}^{l}, \\
\Gamma_{n, p \rightarrow n n}^{l}= & \tilde{\Gamma}_{n, p \rightarrow n n, 111}^{l, n}+\tilde{\Gamma}_{n, p \rightarrow n n, 000}^{l, n}+\tilde{\Gamma}_{n, p \rightarrow n n, 110}^{l, n}-\tilde{\Gamma}_{n, p \rightarrow n n, 101}^{l, n}-\tilde{\Gamma}_{n, p \rightarrow n n, 011}^{l, n} \\
& -\tilde{\Gamma}_{n, p \rightarrow n n, 100}^{l, n}-\tilde{\Gamma}_{n, p \rightarrow n n, 010}^{l, n}+\tilde{\Gamma}_{n, p \rightarrow n n, 001}^{l, n}, \\
\Gamma_{n, p \rightarrow n n}^{u}= & 0, \\
\Gamma_{p, n \rightarrow n n}^{l}= & 0, \\
\Gamma_{p, n \rightarrow n n}^{u}= & 2\left(-\tilde{\Gamma}_{p, n \rightarrow n n, 111}^{u, n}+\tilde{\Gamma}_{p, n \rightarrow n n, 101}^{u, n}-\tilde{\Gamma}_{p, n \rightarrow n n, 011}^{u, n}+\tilde{\Gamma}_{p, n \rightarrow n n, 001}^{u, n}\right), \\
\Gamma_{n, p \rightarrow n p}^{l}= & 0, \\
\Gamma_{n, p \rightarrow n p}^{u}= & \tilde{\Gamma}_{n, p \rightarrow n p, 111}^{u, n}+\tilde{\Gamma}_{n, p \rightarrow n p, 000}^{u, n}+\tilde{\Gamma}_{n, p \rightarrow n p, 110}^{u, n}-\tilde{\Gamma}_{n, p \rightarrow n p, 101}^{u, n}-\tilde{\Gamma}_{n, p \rightarrow n p, 011}^{u, n} \\
& -\tilde{\Gamma}_{n, p \rightarrow n p, 100}^{u, n}-\tilde{\Gamma}_{n, p \rightarrow n p, 010}^{u, n}+\tilde{\Gamma}_{n, p \rightarrow n p, 001}^{u, n}, \\
& +4\left(-\tilde{\Gamma}_{p, p \rightarrow n p, 111}^{l, p}+\tilde{\Gamma}_{p, p \rightarrow n p, 110}^{l, p}\right), \\
\Gamma_{p, n \rightarrow n p}^{l}= & 2\left(-\tilde{\Gamma}_{p, n \rightarrow n p, 111}^{u, n}+\tilde{\Gamma}_{p, n \rightarrow n p, 101}^{u, n}-\tilde{\Gamma}_{p, n \rightarrow n p, 011}^{u, n}+\tilde{\Gamma}_{p, n \rightarrow n p, 001}^{u, n}\right), \\
\Gamma_{p, n \rightarrow n p}^{u}= & 0, \\
\Gamma_{p, p \rightarrow n p}^{l}= & 2\left(-\tilde{\Gamma}_{p, p \rightarrow n p, 111}^{l, n}-\tilde{\Gamma}_{p, p \rightarrow n p, 101}^{l, n}+\tilde{\Gamma}_{p, p \rightarrow n p, 011}^{l, n}+\tilde{\Gamma}_{p, p \rightarrow n p, 001}^{l, n}\right) \\
& +\Gamma_{p, p \rightarrow n p}^{l} .
\end{aligned}
$$

For $\Gamma_{i, i^{\prime} \rightarrow j}^{e d d}$ we have, $\Gamma_{i, i^{\prime} \rightarrow j}^{e d d, l}+\Gamma_{i, i^{\prime} \rightarrow j}^{e d d, u}=\Gamma_{i, i^{\prime} \rightarrow j}^{d d e, l}+\Gamma_{i, i^{\prime} \rightarrow j}^{d d e, u}$. 


\section{A.3. The $\Gamma_{i, i^{\prime} \rightarrow j}^{e d e}$-contribution}

The $\mathcal{S}_{\tau^{\prime} \tau_{N} \tau}^{\text {ede }}\left(q, q^{\prime}, t\right)$ function is

$$
\begin{aligned}
\mathcal{S}_{\tau^{\prime} \tau_{N} \tau}^{\text {de }}\left(q, q^{\prime}, t\right)= & 2 \mathcal{V}_{C, \tau_{N}}\left\{( \hat { \boldsymbol { q } } \cdot \hat { \boldsymbol { q } } ^ { \prime } ) \left(S_{\tau^{\prime}} S_{\tau}+S_{\tau^{\prime}}^{\prime} S_{\tau}^{\prime}+S_{\tau^{\prime}} S_{\tau}^{\prime}+S_{\tau} S_{\tau^{\prime}}^{\prime}+4 S_{V, \tau^{\prime}} S_{V, \tau}\right.\right. \\
& \left.-2\left(S_{\tau^{\prime}} S_{V, \tau}+S_{\tau} S_{V, \tau^{\prime}}+S_{\tau^{\prime}}^{\prime} S_{V, \tau}+S_{\tau}^{\prime} S_{V, \tau^{\prime}}\right)\right)+P_{C, \tau^{\prime}} P_{C, \tau} \\
& +9 P_{\sigma, \tau^{\prime}} P_{\sigma, \tau}+P_{L, \tau^{\prime}} P_{L, \tau}+3\left(P_{\sigma, \tau^{\prime}} P_{C, \tau}+P_{C, \tau^{\prime}} P_{\sigma, \tau}\right)+P_{C, \tau^{\prime}} P_{L, \tau} \\
& \left.+P_{L, \tau^{\prime}} P_{C, \tau}+3\left(P_{\sigma, \tau^{\prime}} P_{L, \tau}+P_{L, \tau^{\prime}} P_{\sigma, \tau}\right)\right\} \\
& +2 \mathcal{V}_{\sigma, \tau_{N}}\left\{( \hat { \boldsymbol { q } } \cdot \hat { \boldsymbol { q } } ^ { \prime } ) \left(3 S_{\tau^{\prime}} S_{\tau}+3 S_{\tau^{\prime}}^{\prime} S_{\tau}^{\prime}-\left(S_{\tau^{\prime}} S_{\tau}^{\prime}+S_{\tau} S_{\tau^{\prime}}^{\prime}\right)+4 S_{V, \tau^{\prime}} S_{V, \tau}\right.\right. \\
& \left.+S_{\tau^{\prime}} S_{V, \tau}+S_{\tau} S_{V, \tau^{\prime}}+S_{\tau^{\prime}}^{\prime} S_{V, \tau}+S_{\tau}^{\prime} S_{V, \tau^{\prime}}\right)+3 P_{C, \tau^{\prime}} P_{C, \tau}+3 P_{\sigma, \tau^{\prime}} P_{\sigma, \tau} \\
& +\left(-1+4\left(\hat{\boldsymbol{q}} \cdot \hat{\boldsymbol{q}}^{\prime}\right)^{2}\right) P_{L, \tau^{\prime}} P_{L, \tau}-3\left(P_{\sigma, \tau^{\prime}} P_{C, \tau}+P_{C, \tau^{\prime}} P_{\sigma, \tau}\right) \\
& \left.-\left(P_{C, \tau^{\prime}} P_{L, \tau}+P_{L, \tau^{\prime}} P_{C, \tau}\right)-\left(P_{\sigma, \tau^{\prime}} P_{L, \tau}+P_{L, \tau^{\prime}} P_{\sigma, \tau}\right)\right\} \\
& +2 \mathcal{V}_{L, \tau_{N}}\left\{\left(\hat{\boldsymbol{q}} \cdot \hat{\boldsymbol{q}}^{\prime}\right)\left(S_{\tau^{\prime}} S_{\tau}+S_{\tau^{\prime}}^{\prime} S_{\tau}^{\prime}\right)\right. \\
& +\left(-\hat{\boldsymbol{q}} \cdot \hat{\boldsymbol{q}}^{\prime}+2(\hat{\boldsymbol{q}} \cdot \hat{\boldsymbol{t}})\left(\hat{\boldsymbol{q}}^{\prime} \cdot \hat{\boldsymbol{t}}\right)\right)\left(S_{\tau^{\prime}} S_{\tau}^{\prime}+S_{\tau} S_{\tau^{\prime}}^{\prime}\right) \\
& +2(\hat{\boldsymbol{q}} \cdot \hat{\boldsymbol{t}})\left(\hat{\boldsymbol{q}}^{\prime} \cdot \hat{\boldsymbol{t}}\right)\left(S_{V, \tau^{\prime}} S_{V, \tau}+S_{\tau^{\prime}} S_{V, \tau}+S_{\tau} S_{V, \tau^{\prime}}+S_{\tau^{\prime}}^{\prime} S_{V, \tau}+S_{\tau}^{\prime} S_{V, \tau^{\prime}}\right) \\
& +P_{C, \tau^{\prime}} P_{C, \tau}+P_{\sigma, \tau^{\prime}} P_{\sigma, \tau}-\left(P_{\sigma, \tau^{\prime}} P_{C, \tau}+P_{C, \tau^{\prime}} P_{\sigma, \tau}\right) \\
& +\left(1+4\left(\hat{\boldsymbol{q}} \cdot \hat{\boldsymbol{q}}^{\prime}\right)(\hat{\boldsymbol{q}} \cdot \hat{\boldsymbol{t}})\left(\hat{\boldsymbol{q}}^{\prime} \cdot \hat{\boldsymbol{t}}\right)-2(\hat{\boldsymbol{q}} \cdot \hat{\boldsymbol{t}})^{2}-2\left(\hat{\boldsymbol{q}}^{\prime} \cdot \hat{\boldsymbol{t}}\right)^{2}\right) P_{L, \tau^{\prime}} P_{L, \tau} \\
& +\left(-1+2\left(\hat{\boldsymbol{t}} \cdot \hat{\boldsymbol{q}}^{\prime}\right)^{2}\right)\left(P_{C, \tau^{\prime}} P_{L, \tau}+P_{L, \tau^{\prime}} P_{C, \tau}\right) \\
& \left.+\left(-1+2(\hat{\boldsymbol{t}} \cdot \hat{\boldsymbol{q}})^{2}\right)\left(P_{\sigma, \tau^{\prime}} P_{L, \tau}+P_{L, \tau^{\prime}} P_{\sigma, \tau}\right)\right\}
\end{aligned}
$$

where $q=p_{1}-h_{i}, t=k-p_{1}$ and $q^{\prime}=p_{1}-h_{i^{\prime}}$.

$$
\begin{aligned}
& \Gamma_{n, n \rightarrow n n}^{l}= \tilde{\Gamma}_{n, n \rightarrow n n, 111}^{l, n}+\tilde{\Gamma}_{n, n \rightarrow n n, 000}^{l, n}+\tilde{\Gamma}_{n, n \rightarrow n n, 110}^{l, n}+\tilde{\Gamma}_{n, n \rightarrow n n, 101}^{l, n}+\tilde{\Gamma}_{n, n \rightarrow n n, 011}^{l, n} \\
&+\tilde{\Gamma}_{n, n \rightarrow n n, 100}^{l, n}+\tilde{\Gamma}_{n, n \rightarrow n n, 010}^{l, n}+\tilde{\Gamma}_{n, n \rightarrow n n, 001}^{l, n}, \\
& \Gamma_{n, n \rightarrow n n}^{u}= \Gamma_{n, n \rightarrow n n}^{l, n}, \\
& \Gamma_{n, p \rightarrow n n}^{l}= 2\left(-\tilde{\Gamma}_{n, p \rightarrow n n, 111}^{l, n}-\tilde{\Gamma}_{n, p \rightarrow n n, 110}^{l, n}+\tilde{\Gamma}_{n, p \rightarrow n n, 101}^{l, n}+\tilde{\Gamma}_{n, p \rightarrow n n, 100}^{l, n}\right), \\
& \Gamma_{n, p \rightarrow n n}^{u}= 0, \\
& \Gamma_{p, n \rightarrow n n}^{l}=0, \\
& \Gamma_{p, n \rightarrow n n}^{u}=2\left(-\tilde{\Gamma}_{p, n \rightarrow n n, 111}^{u, n}+\tilde{\Gamma}_{p, n \rightarrow n n, 101}^{u, n}-\tilde{\Gamma}_{p, n \rightarrow n n, 011}^{u, n}+\tilde{\Gamma}_{p, n \rightarrow n n, 001}^{u, n}\right), \\
& \Gamma_{n, p \rightarrow n p}^{l}=0, \\
& \Gamma_{n, p \rightarrow n p}^{u}=2\left(-\tilde{\Gamma}_{n, p \rightarrow n p, 111}^{u, n}-\tilde{\Gamma}_{n, p \rightarrow n p, 110}^{u, n}+\tilde{\Gamma}_{n, p \rightarrow n p, 101}^{u, n}+\tilde{\Gamma}_{n, p \rightarrow n p, 100}^{u, n}\right), \\
& \Gamma_{p, n \rightarrow n p}^{l}=2\left(-\tilde{\Gamma}_{p, n \rightarrow n p, 111}^{l, n}+\tilde{\Gamma}_{p, n \rightarrow n p, 101}^{l, n}-\tilde{\Gamma}_{p, n \rightarrow n p, 011}^{l, n}+\tilde{\Gamma}_{p, n \rightarrow n p, 001}^{l, n}\right), \\
& \Gamma_{p, n \rightarrow n p}^{u}=0, \\
& \Gamma_{p, p \rightarrow n p}^{l}=2\left(\tilde{\Gamma}_{p, p \rightarrow n p, 111}^{l, n}-\tilde{\Gamma}_{p, p \rightarrow n p, 110}^{l, n}-\tilde{\Gamma}_{p, p \rightarrow n p, 011}^{l, n}+\tilde{\Gamma}_{p, p \rightarrow n p, 010}^{l, n}\right) \\
&+4\left(\tilde{\Gamma}_{p, p \rightarrow n p, 111}^{l, p}+\tilde{\Gamma}_{p, p \rightarrow n p, 101}^{l, p}\right), \\
& \Gamma_{p, p \rightarrow n p}^{u}= \Gamma_{p, p \rightarrow n p}^{l} .
\end{aligned}
$$


A.4. The $\Gamma_{i, i^{\prime} \rightarrow j}^{\text {dee }}$ - and $\Gamma_{i, i^{\prime} \rightarrow j^{-c o n t r i b u t i o n s}}^{e e d}$

The $\mathcal{S}_{\tau^{\prime} \tau_{N} \tau}^{\text {dee }}\left(q, q^{\prime}, t\right)$ function is

$$
\begin{aligned}
\mathcal{S}_{\tau^{\prime} \tau_{N} \tau}^{\text {dee }}\left(q, q^{\prime}, t\right)= & 2 \mathcal{V}_{C, \tau_{N}}\left\{( \hat { \boldsymbol { q } } \cdot \hat { \boldsymbol { q } } ^ { \prime } ) \left(\left(S_{\tau^{\prime}}+S_{\tau}^{\prime}\right)\left(S_{\tau}+S_{\tau^{\prime}}^{\prime}\right)-2\left(S_{\tau} S_{V, \tau^{\prime}}+S_{\tau^{\prime}} S_{V, \tau}\right)\right.\right. \\
& \left.+2\left(S_{\tau}^{\prime} S_{V, \tau^{\prime}}+S_{\tau^{\prime}}^{\prime} S_{V, \tau}\right)\right)+P_{C, \tau^{\prime}} P_{C, \tau}-3 P_{\sigma, \tau^{\prime}} P_{\sigma, \tau} \\
& +\left(2\left(\hat{\boldsymbol{q}} \cdot \hat{\boldsymbol{q}}^{\prime}\right)^{2}-1\right) P_{L, \tau^{\prime}} P_{L, \tau}+3\left(P_{\sigma, \tau^{\prime}} P_{C, \tau}+P_{C, \tau^{\prime}} P_{\sigma, \tau}\right) \\
& \left.+P_{C, \tau^{\prime}} P_{L, \tau}+P_{L, \tau^{\prime}} P_{C, \tau}-\left(P_{\sigma, \tau^{\prime}} P_{L, \tau}+P_{L, \tau^{\prime}} P_{\sigma, \tau}\right)\right\} \\
& +2 \mathcal{V}_{\sigma, \tau_{N}}\left\{( \hat { \boldsymbol { q } } \cdot \hat { \boldsymbol { q } } ^ { \prime } ) \left(3 S_{\tau^{\prime}} S_{\tau}-S_{\tau^{\prime}}^{\prime} S_{\tau}^{\prime}+3 S_{\tau^{\prime}} S_{\tau}^{\prime}-S_{\tau} S_{\tau^{\prime}}^{\prime}\right.\right. \\
& \left.+2 S_{\tau} S_{V, \tau^{\prime}}-6 S_{\tau^{\prime}} S_{V, \tau}-2 S_{\tau}^{\prime} S_{V, \tau^{\prime}}-S_{\tau^{\prime}}^{\prime} S_{V, \tau}\right)+3 P_{C, \tau^{\prime}} P_{C, \tau} \\
& +3 P_{\sigma, \tau^{\prime}} P_{\sigma, \tau}+\left(1-\left(\hat{\boldsymbol{q}} \cdot \hat{\boldsymbol{q}}^{\prime}\right)^{2}\right) P_{L, \tau^{\prime}} P_{L, \tau}+9 P_{C, \tau^{\prime}} P_{\sigma, \tau}-3 P_{\sigma, \tau^{\prime}} P_{C, \tau} \\
& \left.+3 P_{C, \tau^{\prime}} P_{L, \tau}-P_{L, \tau^{\prime}} P_{C, \tau}+P_{\sigma, \tau^{\prime}} P_{L, \tau}+P_{L, \tau^{\prime}} P_{\sigma, \tau}\right\} \\
& +2 \mathcal{V}_{L, \tau}\left\{\left(\hat{\boldsymbol{q}} \cdot \hat{\boldsymbol{q}}^{\prime}\right)\left(S_{\tau^{\prime}} S_{\tau}+S_{\tau^{\prime}} S_{\tau}^{\prime}-2 S_{\tau^{\prime}} S_{V, \tau}\right)\right. \\
& +\left(-\hat{\boldsymbol{q}} \cdot \hat{\boldsymbol{q}}^{\prime}+2(\hat{\boldsymbol{q}} \cdot \hat{\boldsymbol{t}})\left(\hat{\boldsymbol{q}}^{\prime} \cdot \hat{\boldsymbol{t}}\right)\right)\left(S_{\tau^{\prime}}^{\prime} S_{\tau}^{\prime}+S_{\tau} S_{\tau^{\prime}}^{\prime}-2 S_{\tau^{\prime}}^{\prime} S_{V, \tau}\right) \\
& +(\hat{\boldsymbol{q}} \cdot \hat{\boldsymbol{t}})\left(\hat{\boldsymbol{q}}^{\prime} \cdot \hat{\boldsymbol{t}}\right)\left(S_{\tau} S_{V, \tau^{\prime}}-S_{\tau}^{\prime} S_{V, \tau^{\prime}}\right)+P_{C, \tau^{\prime}} P_{C, \tau}+P_{\sigma, \tau^{\prime}} P_{\sigma, \tau} \\
& +\left(1+4\left(\hat{\boldsymbol{q}} \cdot \hat{\boldsymbol{q}}^{\prime}\right)(\hat{\boldsymbol{q}} \cdot \hat{\boldsymbol{t}})\left(\hat{\boldsymbol{q}}^{\prime} \cdot \hat{\boldsymbol{t}}\right)-2(\hat{\boldsymbol{q}} \cdot \hat{\boldsymbol{t}})^{2}-2\left(\hat{\boldsymbol{q}}^{\prime} \cdot \hat{\boldsymbol{t}}\right)^{2}\right) P_{L, \tau^{\prime}} P_{L, \tau} \\
& +3 P_{C, \tau^{\prime}} P_{\sigma, \tau}-P_{\sigma, \tau^{\prime}} P_{C, \tau}+P_{C, \tau^{\prime}} P_{L, \tau}-\left(1-2(\hat{\boldsymbol{q}} \cdot \hat{\boldsymbol{t}})^{2}\right) P_{L, \tau^{\prime}} P_{C, \tau} \\
& \left.+\left(-1+4\left(\hat{\boldsymbol{q}}^{\prime} \cdot \hat{\boldsymbol{t}}\right)^{2}\right) P_{\sigma, \tau^{\prime}} P_{L, \tau}+\left(1-2(\hat{\boldsymbol{q}} \cdot \hat{\boldsymbol{t}})^{2}\right) P_{L, \tau^{\prime}} P_{\sigma, \tau}\right\},
\end{aligned}
$$

where $q=p_{1}-h_{i}, t=h_{i}-h_{i^{\prime}}$ and $q^{\prime}=k-p_{1}$.

$$
\begin{aligned}
\Gamma_{n, n \rightarrow n n}^{l}= & \tilde{\Gamma}_{n, n \rightarrow n n, 111}^{l, n}+\tilde{\Gamma}_{n, n \rightarrow n n, 000}^{l, n}+\tilde{\Gamma}_{n, n \rightarrow n n, 110}^{l, n}+\tilde{\Gamma}_{n, n \rightarrow n n, 101}^{l, n}+\tilde{\Gamma}_{n, n \rightarrow n n, 011}^{l, n} \\
& +\tilde{\Gamma}_{n, n \rightarrow n n, 100}^{l, n}+\tilde{\Gamma}_{n, n \rightarrow n n, 010}^{l, n}+\tilde{\Gamma}_{n, n \rightarrow n n, 001}^{l, n}, \\
\Gamma_{n, n \rightarrow n n}^{u}= & \Gamma_{n, n \rightarrow n n}^{l}, \\
\Gamma_{n, p \rightarrow n n}^{l}= & 2\left(-\tilde{\Gamma}_{n, p \rightarrow n n, 111}^{l, n}-\tilde{\Gamma}_{n, p \rightarrow n n, 110}^{l, n}+\tilde{\Gamma}_{n, p \rightarrow n n, 011}^{l, n}+\tilde{\Gamma}_{n, p \rightarrow n n, 010}^{l, n}\right), \\
\Gamma_{n, p \rightarrow n n}^{u}= & 0, \\
\Gamma_{p, n \rightarrow n n}^{l}= & 0, \\
\Gamma_{p, n \rightarrow n n}^{u}= & 4\left(\tilde{\Gamma}_{p, n \rightarrow n n, 111}^{u, n}+\tilde{\Gamma}_{p, n \rightarrow n n, 011}^{u, n}\right), \\
\Gamma_{n, p \rightarrow n p}^{l}= & 0, \\
\Gamma_{n, p \rightarrow n p}^{u}= & 2\left(-\tilde{\Gamma}_{n, p \rightarrow n p, 111}^{u, n}-\tilde{\Gamma}_{n, p \rightarrow n p, 110}^{u, n}+\tilde{\Gamma}_{n, p \rightarrow n p, 011}^{u, n}+\tilde{\Gamma}_{n, p \rightarrow n p, 010}^{u, n}\right), \\
\Gamma_{p, n \rightarrow n p}^{l}= & 4\left(\tilde{\Gamma}_{p, n \rightarrow n p, 111}^{l, n}+\tilde{\Gamma}_{p, n \rightarrow n p, 011}^{l, n}\right), \\
\Gamma_{p, n \rightarrow n p}^{u}= & 0, \\
\Gamma_{p, p \rightarrow n p}^{l}= & 2\left(-\tilde{\Gamma}_{p, p \rightarrow n p, 111}^{l, n}-\tilde{\Gamma}_{p, p \rightarrow n p, 101}^{l, n}+\tilde{\Gamma}_{p, p \rightarrow n p, 011}^{l, n}+\tilde{\Gamma}_{p, p \rightarrow n p, 001}^{l, n}\right) \\
& +2\left(\tilde{\Gamma}_{p, p \rightarrow n p, 111}^{l, p}-\tilde{\Gamma}_{p, p \rightarrow n p, 110}^{l, p}-\tilde{\Gamma}_{p, p \rightarrow n p, 101}^{l, p}+\tilde{\Gamma}_{p, p \rightarrow n p, 100}^{l, p}\right), \\
\Gamma_{p, p \rightarrow n p}^{u}= & \Gamma_{p, p \rightarrow n p}^{l} .
\end{aligned}
$$

For $\Gamma_{i, i^{\prime} \rightarrow j}^{e e d}$ we have, $\Gamma_{i, i^{\prime} \rightarrow j}^{e e d, l}+\Gamma_{i, i^{\prime} \rightarrow j}^{e e d, u}=\Gamma_{i, i^{\prime} \rightarrow j}^{\text {dee,l}}+\Gamma_{i, i^{\prime} \rightarrow j}^{\text {dee, } u}$. 


\section{A.5. The $\Gamma_{i, i^{\prime} \rightarrow j}^{e e e}$-contribution}

The $\mathcal{S}_{\tau^{\prime} \tau_{N} \tau}^{e e e}\left(q, q^{\prime}, t\right)$ function is

$$
\begin{aligned}
\mathcal{S}_{\tau^{\prime} \tau_{N} \tau}^{e e e}\left(q, q^{\prime}, t\right)= & 4 \mathcal{V}_{C, \tau_{N}}\left\{\left(\hat{\boldsymbol{q}} \cdot \hat{\boldsymbol{q}}^{\prime}\right)\left(S_{\tau^{\prime}} S_{\tau}+S_{\tau^{\prime}}^{\prime} S_{\tau}^{\prime}+2 S_{V, \tau^{\prime}} S_{V, \tau}\right)+P_{C, \tau^{\prime}} P_{C, \tau}\right. \\
& \left.+3 P_{\sigma, \tau^{\prime}} P_{\sigma, \tau}+\left(\hat{\boldsymbol{q}} \cdot \hat{\boldsymbol{q}}^{\prime}\right)^{2} P_{L, \tau^{\prime}} P_{L, \tau}+P_{\sigma, \tau^{\prime}} P_{L, \tau}+P_{L, \tau^{\prime}} P_{\sigma, \tau}\right\} \\
& +4 \mathcal{V}_{\sigma, \tau_{N}}\left\{\left(\hat{\boldsymbol{q}} \cdot \hat{\boldsymbol{q}}^{\prime}\right)\left(S_{\tau^{\prime}} S_{\tau}^{\prime}+S_{\tau^{\prime}}^{\prime} S_{\tau}-\left(S_{\tau^{\prime}}+S_{\tau}^{\prime}\right)\left(S_{V, \tau^{\prime}}+S_{V, \tau}\right)\right)\right. \\
& +6 P_{\sigma, \tau^{\prime}} P_{\sigma, \tau}+\left(1-\left(\hat{\boldsymbol{q}} \cdot \hat{\boldsymbol{q}}^{\prime}\right)^{2}\right) P_{L, \tau^{\prime}} P_{L, \tau}+3\left(P_{C, \tau^{\prime}} P_{\sigma, \tau}+P_{\sigma, \tau^{\prime}} P_{C, \tau}\right) \\
& \left.+P_{C, \tau^{\prime}} P_{L, \tau}+P_{L, \tau^{\prime}} P_{C, \tau}+2\left(P_{\sigma, \tau^{\prime}} P_{L, \tau}+P_{L, \tau^{\prime}} P_{\sigma, \tau}\right)\right\} \\
& +4 \mathcal{V}_{L, \tau_{N}}\left\{(\hat{\boldsymbol{q}} \cdot \hat{\boldsymbol{t}})\left(\hat{\boldsymbol{q}}^{\prime} \cdot \hat{\boldsymbol{t}}\right)\left(S_{\tau^{\prime}} S_{\tau}^{\prime}+S_{\tau^{\prime}}^{\prime} S_{\tau}+2 S_{V, \tau^{\prime}} S_{V, \tau}\right)\right. \\
& -\left(\left(\hat{\boldsymbol{q}} \cdot \hat{\boldsymbol{q}}^{\prime}\right)-(\hat{\boldsymbol{q}} \cdot \hat{\boldsymbol{t}})\left(\hat{\boldsymbol{q}}^{\prime} \cdot \hat{\boldsymbol{t}}\right)\right)\left(S_{\tau^{\prime}}+S_{\tau}^{\prime}\right)\left(S_{V, \tau^{\prime}}+S_{V, \tau}\right)+2 P_{\sigma, \tau^{\prime}} P_{\sigma, \tau} \\
& +\left(1-(\hat{\boldsymbol{q}} \cdot \hat{\boldsymbol{q}})(\hat{\boldsymbol{q}} \cdot \hat{\boldsymbol{t}})\left(\hat{\boldsymbol{q}}^{\prime} \cdot \hat{\boldsymbol{t}}\right)-\left(\hat{\boldsymbol{q}} \cdot \hat{\boldsymbol{q}}^{\prime}\right)^{2}-(\hat{\boldsymbol{q}} \cdot \hat{\boldsymbol{t}})^{2}-\left(\hat{\boldsymbol{q}}^{\prime} \cdot \hat{\boldsymbol{t}}\right)^{2}\right) P_{L, \tau^{\prime}} P_{L, \tau} \\
& +P_{C, \tau^{\prime}} P_{\sigma, \tau}+P_{\sigma, \tau^{\prime}} P_{C, \tau}+\left(\hat{\boldsymbol{q}}^{\prime} \cdot \hat{\boldsymbol{t}}\right)^{2} P_{C, \tau^{\prime}} P_{L, \tau}+(\hat{\boldsymbol{q}} \cdot \hat{\boldsymbol{t}})^{2} P_{L, \tau^{\prime}} P_{C, \tau} \\
& \left.+\left(1-\left(\hat{\boldsymbol{q}}^{\prime} \cdot \hat{\boldsymbol{t}}\right)^{2}\right) P_{\sigma, \tau^{\prime}} P_{L, \tau}+\left(1-(\hat{\boldsymbol{q}} \cdot \hat{\boldsymbol{t}})^{2}\right) P_{L, \tau^{\prime}} P_{\sigma, \tau}\right\},
\end{aligned}
$$

where $q=p_{1}-h_{i}, t=h_{i}-h_{i^{\prime}}$ and $q^{\prime}=p_{1}-h_{i^{\prime}}$.

$$
\begin{aligned}
\Gamma_{n, n \rightarrow n n}^{l}= & \tilde{\Gamma}_{n, n \rightarrow n n, 111}^{l, n}+\tilde{\Gamma}_{n, n \rightarrow n n, 000}^{l, n}+\tilde{\Gamma}_{n, n \rightarrow n n, 110}^{l, n}+\tilde{\Gamma}_{n, n \rightarrow n n, 101}^{l, n}+\tilde{\Gamma}_{n, n \rightarrow n n, 011}^{l, n} \\
& +\tilde{\Gamma}_{n, n \rightarrow n n, 100}^{l, n}+\tilde{\Gamma}_{n, n \rightarrow n n, 010}^{l, n}+\tilde{\Gamma}_{n, n \rightarrow n n, 001}^{l, n}, \\
\Gamma_{n, n \rightarrow n n}^{u}= & \Gamma_{n, n \rightarrow n n}^{l}, \\
\Gamma_{n, p \rightarrow n n}^{l}= & 4\left(\tilde{\Gamma}_{n, p \rightarrow n n, 111}^{l, n}+\tilde{\Gamma}_{n, p \rightarrow n n, 110}^{l, n}\right), \\
\Gamma_{n, p \rightarrow n n}^{u}= & 0, \\
\Gamma_{p, n \rightarrow n n}^{l}= & 0, \\
\Gamma_{p, n \rightarrow n n}^{u}= & 4\left(\tilde{\Gamma}_{p, n \rightarrow n n, 111}^{u, n}+\tilde{\Gamma}_{p, n \rightarrow n n, 011}^{u, n}\right), \\
\Gamma_{n, p \rightarrow n p}^{l}= & 0, \\
\Gamma_{n, p \rightarrow n p}^{u}= & 4\left(\tilde{\Gamma}_{n, p \rightarrow n p, 111}^{u, n}+\tilde{\Gamma}_{n, p \rightarrow n p, 110}^{u, n}\right), \\
\Gamma_{p, n \rightarrow n p}^{l}= & 4\left(\tilde{\Gamma}_{p, n \rightarrow n p, 111}^{l, n}+\tilde{\Gamma}_{p, n \rightarrow n p, 011}^{l, n}\right), \\
\Gamma_{p, n \rightarrow n p}^{u}= & 0, \\
\Gamma_{p, p \rightarrow n p}^{l}= & -\tilde{\Gamma}_{p, p \rightarrow n p, 111}^{l, n}+\tilde{\Gamma}_{p, p \rightarrow n p, 000}^{l, n}+\tilde{\Gamma}_{p, p \rightarrow n p, 110}^{l, n}+\tilde{\Gamma}_{p, p \rightarrow n p, 101}^{l, n} \\
& +\tilde{\Gamma}_{p, p \rightarrow n p, 011}^{l, n}-\tilde{\Gamma}_{p, p \rightarrow n p, 100}^{l, n}-\tilde{\Gamma}_{p, p \rightarrow n p, 010}^{l, n}-\tilde{\Gamma}_{p, p \rightarrow n p, 001}^{l, n} \\
& +4\left(\tilde{\Gamma}_{p, p \rightarrow n p, 111}^{l, p}+\tilde{\Gamma}_{p, p \rightarrow n p, 110}^{l, p}\right), \\
\Gamma_{p, p \rightarrow n p}^{u, p}= & \Gamma_{p, p \rightarrow n p}^{l} .
\end{aligned}
$$




\section{References}

[1] E. Oset, A. Ramos, Prog. Part. Nucl. Phys. 41 (1998) 191.

[2] W.M. Alberico, G. Garbarino, Phys. Rep. 369 (2002) 1;

W.M. Alberico, G. Garbarino, in: T. Bressani, A. Filippi, U. Wiedner (Eds.), Hadron Physics, Proceedings of the International School of Physics "Enrico Fermi", Course CLVIII, Varenna, Italy, 22 June-2 July 2004, IOS Press, Amsterdam, 2005, p. 125.

[3] A. Montwill, et al., Nucl. Phys. A 234 (1974) 413.

[4] O. Hashimoto, et al., Phys. Rev. Lett. 88 (2002) 042503.

[5] Y. Sato, et al., Phys. Rev. C 71 (2005) 025203.

[6] J.H. Kim, et al., Phys. Rev. C 68 (2003) 065201.

[7] S. Okada, et al., Phys. Lett. B 597 (2004) 249.

[8] H. Bhang, in: F. Anulli, M. Bertani, G. Capon, C. Curceanu-Petrascu, F.L. Fabbri, S. Miscetti (Eds.), DAPHNE 2004: Physics at Meson Factories, Frascati Phys. Ser. 36 (2005) 243.

[9] H. Outa, in: T. Bressani, A. Filippi, U. Wiedner (Eds.), Hadron Physics, IOS Press, Amsterdam, 2005, p. 219; H. Outa, et al., Nucl. Phys. A 754 (2005) 157c.

[10] B.H. Kang, et al., Phys. Rev. Lett. 96 (2006) 062301.

[11] M.J. Kim, et al., Phys. Lett. B 641 (2006) 28.

[12] W.M. Alberico, G. Garbarino, A. Parreño, A. Ramos, Phys. Rev. Lett. 94 (2005) 082501.

[13] C. Chumillas, G. Garbarino, A. Parreño, A. Ramos, nucl-th/0705.0231.

[14] J.B. Adams, Phys. Rev. 156 (1967) 1611.

[15] B.H.J. McKellar, B.F. Gibson, Phys. Rev. C 30 (1984) 322.

[16] T. Motoba, K. Itonaga, Prog. Theor. Phys. Suppl. 117 (1994) 477.

[17] A. Parreño, A. Ramos, C. Bennhold, Phys. Rev. C 52 (1995) R1768;

A. Parreño, A. Ramos, C. Bennhold, Phys. Rev. C 54 (1996) 1500.

[18] J.F. Dubach, G.B. Feldman, B.R. Holstein, L. de la Torre, Ann. Phys. 249 (1996) 146.

[19] A. Parreño, A. Ramos, C. Bennhold, Phys. Rev. C 56 (1997) 339.

[20] A. Parreño, A. Ramos, N.G. Kelkar, C. Bennhold, Phys. Rev. C 59 (1999) 2122.

[21] D. Jido, E. Oset, J.E. Palomar, Nucl. Phys. A 694 (2001) 525.

[22] A. Parreño, A. Ramos, Phys. Rev. C 65 (2002) 015204.

[23] K. Itonaga, T. Ueda, T. Motoba, Phys. Rev. C 65 (2002) 034617.

[24] E. Bauer, F. Krmpotić, Nucl. Phys. A 717 (2003) 217.

[25] K. Maltman, M. Shmatikov, Phys. Lett. B 331 (1994) 1.

[26] J. Golak, K. Miyagawa, H. Kamada, H. Witala, W. Glöckle, A. Parreño, A. Ramos, C. Bennhold, Phys. Rev. C 55 (1997) 2196;

J. Golak, K. Miyagawa, H. Kamada, H. Witala, W. Glöckle, A. Parreño, A. Ramos, C. Bennhold, Phys. Rev. C 56 (1997) 2892, Erratum.

[27] A. Parreño, A. Ramos, C. Bennhold, Maltman, Phys. Lett. B 435 (1998) 1.

[28] C.Y. Cheung, D.P. Heddle, L.S. Kisslinger, Phys. Rev. C 27 (1983) 335.

[29] D.P. Heddle, L.S. Kisslinger, Phys. Rev. C 33 (1986) 608.

[30] K. Maltman, M. Shmatikov, Phys. Lett. B 331 (1994) 1;

K. Maltman, M. Shmatikov, Nucl. Phys. A 585 (1995) 343c.

[31] T. Inoue, S. Takeuchi, M. Oka, Nucl. Phys. A 577 (1994) 281c;

T. Inoue, S. Takeuchi, M. Oka, Nucl. Phys. A 597 (1996) 563;

T. Inoue, M. Oka, T. Motoba, K. Itonaga, Nucl. Phys. A 633 (1998) 312;

T. Inoue, M. Oka, T. Motoba, K. Itonaga, Nucl. Phys. A 633 (1998) 312.

[32] K. Sasaki, T. Inoue, M. Oka, Nucl. Phys. A 669 (2000) 331;

K. Sasaki, T. Inoue, M. Oka, Nucl. Phys. A 678 (2000) 455, Erratum;

K. Sasaki, T. Inoue, M. Oka, Nucl. Phys. A 707 (2002) 477.

[33] W.M. Alberico, A. De Pace, M. Ericson, A. Molinari, Phys. Lett. B 256 (1991) 134.

[34] A. Ramos, E. Oset, L.L. Salcedo, Phys. Rev. C 50 (1994) 2314.

[35] A. Ramos, M.J. Vicente-Vacas, E. Oset, Phys. Rev. C 55 (1997) 735; A. Ramos, M.J. Vicente-Vacas, E. Oset, Phys. Rev. C 66 (2002) 039903, Erratum.

[36] W.M. Alberico, A. De Pace, G. Garbarino, A. Ramos, Phys. Rev. C 61 (2000) 044314.

[37] W.M. Alberico, A. De Pace, G. Garbarino, R. Cenni, Nucl. Phys. A 668 (2000) 113.

[38] E. Bauer, F. Krmpotić, Nucl. Phys. A 739 (2004) 109. 
[39] G. Garbarino, A. Parreño, A. Ramos, Phys. Rev. Lett. 91 (2003) 112501.

[40] G. Garbarino, A. Parreño, A. Ramos, Phys. Rev. C 69 (2004) 054603.

[41] E. Bauer, G. Garbarino, A. Parreño, A. Ramos, nucl-th/0602066.

[42] E. Bauer, Nucl. Phys. A 781 (2007) 424.

[43] E. Oset, L.L. Salcedo, Nucl. Phys. A 443 (1985) 704.

[44] E. Bauer, A. Ramos, A. Polls, Phys. Rev. C 54 (1996) 2959.

[45] M.N. Nagels, T.A. Rijiken, J.J. de Swart, Phys. Rev. D 15 (1977) 2547;

P.M.M. Maessen, T.A. Rijiken, J.J. de Swart, Phys. Rev. C 40 (1989) 2226.

[46] R. Machleidt, K. Holinde, Ch. Elster, Phys. Rep. 149 (1987) 1.

[47] M.B. Barbaro, A. De Pace, T.W. Donnelly, A. Molinari, Nucl. Phys. A 596 (1996) 553. 